medRxiv preprint doi: https://doi.org/10.1101/2020.02.24.20027532; this version posted February 27, 2020. The copyright holder for this preprint

(which was not certified by peer review) is the author/funder, who has granted medRxiv a license to display the preprint in perpetuity.

It is made available under a CC-BY-ND 4.0 International license .

\title{
A systematic review and meta-analysis considering the SSRI, SNRI and TCA classes of antidepressants and the risk for congenital heart defects
}

Courtney De Vries, Svetla Gadzhanova, Matthew J. Sykes, Michael Ward, Elizabeth Roughead

\begin{abstract}
Background Antidepressant use during the first trimester is reported in $4 \%$ to $8 \%$ of pregnancies. The use of some selective serotonin reuptake inhibitors (SSRI) during this stage of gestation has been identified as increasing the odds for congenital heart defects, however little is known about the safety of non-SSRI antidepressants.
\end{abstract}

Objective To assess the odds of congenital heart defects associated with the use of any antidepressant during the first trimester of pregnancy. To investigate individual classes of antidepressants: SSRIs, serotonin norepinephrine reuptake inhibitors (SNRI), tricyclic antidepressants (TCA) and individual antidepressants.

Data sources PubMed and Embase were searched without restrictions from inception till 2 January 2020.

Study selection Prospective and retrospective cohort and case-control studies were included if they documented the maternal usage of antidepressants during the first trimester of pregnancy and assessed the presence of congenital heart defects.

Data extraction and meta-analysis Data were extracted by two independent reviewers and the endpoint assessed was congenital heart defects. Where studies reported multiple results for different types of heart defects or individual antidepressants, results were combined when possible. Analyses assessing individual antidepressants and classes of antidepressants (SSRIs, SNRIs and TCAs) were undertaken.

Results A total of 16 studies were identified, encompassing 4,564,798 pregnancy outcomes. The odds ratio for maternal use of any antidepressant and the presence of congenital heart defects from the mixed-methods meta-analysis was 1.22 (95\% confidence interval ( $\mathrm{Cl}$ ): 1.11 to 1.33 ).

Analyses of antidepressants by class produced an odds ratio of 1.50 (95\% Cl: 1.19 to 1.89 ) for maternal SNRI use during the first trimester of pregnancy and the formation of congenital heart defects. A significant odds ratio of 1.22 (95\% Cl: 1.12 to 1.33 ) was reported for SSRIs. For the TCA class, no increased odds ratio was found.

Analyses of individual antidepressants produced significant odds ratios of 1.53 ( $95 \% \mathrm{Cl}: 1.25$ to 1.88 ), 1.28 (95\% Cl: 1.01 to 1.62 ), 1.28 (95\% Cl: 1.14 to 1.45 ) and 1.23 (95\% Cl: 1.01 to 1.50) for paroxetine, fluoxetine, sertraline and bupropion respectively.

Conclusion While some insight has been gained into which classes of antidepressant and individual antidepressants pose more risk than others for causing congenital heart defects, information regarding some antidepressants is still lacking.

\section{Introduction}

Antidepressants are used during pregnancy, with the prevalence of maternal antidepressant use increasing over time. ${ }^{1-3}$ In Denmark, the use of selective serotonin reuptake inhibitors (SSRI) in pregnancy increased from approximately $0.5 \%$ to $3 \%$ between 2000 and $2010 .{ }^{1}$ In the United States of America (USA), the Birth Defects Study identified that the use of any antidepressant during the first trimester of pregnancy increased from less than $0.5 \%$ before 1987 , to $3 \%$ in 1999 and $8 \%$ in $2008 .{ }^{2}$ This study identified that use of selective serotonin reuptake inhibitors (SSRI) was $2 \%$ in 1999 and $6 \%$ in 2008. ${ }^{2}$ Dispensing records between 2006 and 2011 in the USA, identified the use of any antidepressant and SSRIs during the first trimester of pregnancy as $5 \%$ and $4 \%$ respectively. ${ }^{3}$ 
Paroxetine was the first SSRI to reach the American market in 1992. In 2005, an alert was released stating that the use of paroxetine during pregnancy had been associated with an increased risk of major congenital malformations, especially those relating to the heart. ${ }^{4} \mathrm{~A} 2016$ meta-analysis reported an odds ratio of 1.23 (95\% confidence interval $(\mathrm{Cl}): 1.06$ to 1.43 ) for paroxetine use during the first trimester of pregnancy and any cardiac malformations. ${ }^{5}$ Specific cardiac defects were also investigated in this metaanalysis and some were reported to be significant, including: atrial septal defects, right ventricular outflow tract obstructions, bulbus cordis anomalies and anomalies of cardiac septal closure. ${ }^{5}$

Medications from the same class may exert similar toxicities, meaning that other antidepressants may also increase the risk for congenital heart defects. It is desirable to minimise medicine use in pregnancy but discontinuing antidepressant therapy in unwell women may be complicated by the risks of suicidal ideation, relapse of depression and anxiety or panic attacks. ${ }^{67}$ The severity of these risks mean that it may not be possible to cease antidepressant therapy. Evidence regarding the safety of antidepressant use in human pregnancies is required for all women.

Pharmacoepidemiological studies provide data for birth outcomes following antidepressant use during the first trimester of pregnancy, which is the relevant period to investigate for cardiac defects as the heart is formed during weeks 4 to 8 of gestation. Previous meta-analyses have revealed significant relationships between usage of paroxetine, ${ }^{589}$ fluoxetine $^{10}{ }^{11}$ sertraline $^{12}$ and any antidepressant ${ }^{13}$ during the first trimester of pregnancy and an increased formation of heart defects. Only one of these meta-analyses considered non-SSRI antidepressants, ${ }^{13}$ and several cohort and case-control studies have been published since the last published meta-analysis. ${ }^{14-18}$ The largest meta-analysis to date was carried out by Shen et al. ${ }^{12}$ and investigated the first trimester use of sertraline and congenital heart defects. This meta-analysis included 12 cohort studies with a total of 6,468,241 pregnant women and found a statistically significant increased risk of cardiovascular defects, with an odds ratio of 1.36 (95\% Cl: 1.06 to 1.74$)$.

The aim of this systematic literature review and meta-analysis was to investigate the relationship between usage of any antidepressant during the first trimester of pregnancy and the development of congenital heart defects. An investigation into specific classes of antidepressants was also completed to assess whether a class-effect exists between first trimester antidepressant use and congenital heart defects. The relationship between usage of specific antidepressants during the first trimester of pregnancy and heart defects was also investigated.

\section{Methods}

\section{Literature review}

A literature review was completed using PubMed and Embase. The databases were searched from inception to 2 January 2020 using MeSH terms listed in supplement 1. Search terms were searched independently and then combined within each database to retrieve articles reporting the use of antidepressive medications in pregnancy and adverse outcomes. The search strategy and results are detailed in supplement 1 . The results were limited to human data and no further limits were imposed on the search. Duplicates were removed before moving onto the title and abstract reviews. PRISMA guidelines were followed for all procedures and reporting (supplement 2).

\section{Study selection criteria and data extraction}

The title and abstract inclusion criteria were cohort or case-control studies with a comparison group of women who had not used antidepressants during pregnancy, study participants aged 15 years or older, maternal use of antidepressants during the first trimester of pregnancy, and outcomes which included 
congenital heart defects or other malformations. Live births, stillbirths and terminations of pregnancy were all classified as suitable endpoints of pregnancies. If the usage of antidepressant agents was not confirmed, repeated prescriptions or dispensations of an agent were assumed to correlate directly to maternal usage.

The title and abstract exclusion criteria were: reviews, study protocols, case series, case reports, editorials, commentaries, letters to the editor, conference papers, study participants under 15 years of age, studies which contained only abortive, mortal and neurobehavioral outcomes and if the data collected did not pertain to antidepressant medications. The form for title and abstract reviews is provided in supplement 3.

The quality of eligible studies was assessed using a modified version of the Newcastle-Ottawa quality assessment scale. While the Newcastle Ottawa Scale assesses important study features it is not intuitive to use and reviews have documented that there is a low inter-reviewer reliability and poor agreement about study quality between authors and reviewers when using this scale. ${ }^{19} 20$ For this reason the scale was altered to make it tailored for assessing congenital heart defects as the outcome of interest and to improve categorisation of study design. To improve the ease of which reviewers could assess study design the scale was converted to a tabular format which allowed for an analysis on the study quality to be compiled into a single document. The advantage of this method is that it allowed customisation of the documentation of the study methods before grouping the criteria into high, medium or low categories and the assessment outcomes into ideal, good, acceptable or poor outcomes (supplement 4). High impact criteria assessed the selection of study participants and the determination of outcomes and antidepressant use. Follow-up was classified as being a medium impact criterion. Low impact criteria assessed other study features including maternal smoking, alcohol or medication use and whether the non-medicated comparison group was depressed or not.

If three of the high impact criteria were ideal, the end of follow-up was not poor and at least one of the low impact criteria were ideal for cohort studies, the study was included. Cohort studies were excluded if any of the high or medium impact criteria were poor. If four of the high impact criteria were ideal and at least two of the low impact criteria were ideal for case-control studies, the study was included. Casecontrol studies were excluded if any of the high impact criteria were poor. The differences in cohort and case-control study designs produced slightly different study criteria and therefore inclusion decisions. The method for ascertaining antidepressant use, a high impact criterion, was completed for both cohort and case-control studies. For case-control studies an additional criterion was added to confirm that the same method of ascertainment was used for cases and controls. End of follow-up, a medium impact criterion, was only completed for cohort studies to assess pregnancy and birth outcomes. Non-response rate, a low impact criterion, was only completed for case-control studies to identify any differences between the non-response rates of cases and controls (supplement 4).

CDV and SG independently undertook data extraction and quality ranking. Any conflicts were discussed and reviewed by ER.

Once potentially suitable studies were identified, the data sources, study years and settings were compared to ensure that multiple studies did not use the same data. If data sources such as birth registries overlapped between studies, then the most recent study providing the largest sample size was retained. 
All reported crude odds ratios and adjusted odds ratios were extracted from the studies. Additionally, the data needed to calculate odds ratios for antidepressant usage during the first trimester of pregnancy and the formation of congenital heart defects were retrieved from the studies.

Most studies included a single comparison group which had not used antidepressants during pregnancy, however some studies reported multiple comparison groups. Ban, et al. $2014^{14}$ provided data on two unmedicated groups, either depressed or non-depressed. The comparison group in the meta-analysis comprises the sum of unmedicated depressed and non-depressed women. Furu, et al $2015^{16}$ provided data on a full study cohort and a sub-cohort comprised of sibling pairs. Data from the full cohort were included. Davis et al. $2007^{21}$ reported data on SSRIs and tricyclic antidepressants (TCA), however these could not be combined for the primary analysis because the total number of women in the unmedicated comparison group was inconsistent. Therefore, these data are reported individually in all analyses.

Some studies assessed individual heart defects or individual antidepressants. Whenever possible, results from the individual antidepressants and heart defects were aggregated to estimate overall harm for the "any antidepressant" analysis. Classes of antidepressant and individual antidepressants were analysed if data for the meta-analyses were available from at least three studies for each class or antidepressant.

Confounding factors reported in the studies including the use of other medications, maternal smoking and maternal diabetes were recorded.

\section{Statistical analyses}

RevMan $5.3^{22}$ was utilised to perform the meta-analyses. The primary analysis investigated the relationship between maternal use of any antidepressant during the first trimester of pregnancy and the presence of congenital heart defects. The antidepressant classes were stratified into SSRI, serotoninnorepinephrine reuptake inhibitor (SNRI) and TCA groups. Antidepressants were investigated individually, and the results were also stratified by cohort and case-control studies.

Mixed-effects models were used for all meta-analyses because they are more suited to dealing with heterogenous data. An overall odds ratio with a $95 \% \mathrm{Cl}$ measuring the relationship between first trimester antidepressant usage and the presence of heart defects was calculated in all analyses and the significance of these relationships were investigated by the $p$ value for overall effect. Heterogeneity was measured by the $\mathrm{I}^{2}$ values and their corresponding $\mathrm{p}$ values and analyses were defined as being heterogenous if their $p$ values were significant $(<0.05)$.

Outlying studies were defined as having a markedly different intervention effect estimate or an abnormally large confidence interval. To complete a sensitivity analysis, the primary meta-analysis was investigated for outliers and a funnel plot was examined to identify outlying studies (supplement 5 ). The funnel plot is also a small-study effect test. The Mantel-Haenszel mixed-effects meta-analysis was applied, both with and without the outlying studies, to determine the effect of outliers on the overall significance of the results and the heterogeneity.

\section{Results}

Figure 1 shows the article selection process. Following the title and abstract screening, 76 articles were eligible for a full text review. 16 studies, comprising 4,564,798 pregnancy outcomes met the final inclusion criteria and were included. 
An overview of the characteristics of the included studies and detailed information on relevant medication use is reported in table 1. Twelve studies were cohort studies ${ }^{514162123-29}$ and four studies were case controls. ${ }^{18} 30-32$ Eight of the studies were prospective ${ }^{14-16} 1824272833$ and eight were retrospective. ${ }^{21} 232526$ 29-32 For two studies the comparison group included depressed women who were unmedicated. ${ }^{1415}$

The unadjusted and adjusted odds ratios from individual studies are provided in table 2, with significant odds ratios shown in bold text. 
Table 1 Overview of studies included in the systematic review and meta-analyses

\begin{tabular}{|c|c|c|c|c|c|c|}
\hline $\begin{array}{l}\text { Author, Year } \\
\text { Published }\end{array}$ & Study Type & $\begin{array}{l}\text { Study Years } \\
\text { (Setting) }\end{array}$ & End Of Follow Up & $\begin{array}{l}\text { Definition Of Maternal } \\
\text { Antidepressant Use }\end{array}$ & $\begin{array}{l}\text { Definition Of Non- } \\
\text { Antidepressant Use }\end{array}$ & $\begin{array}{l}\text { Proportion Of Cohort With } \\
\text { Concomitant Medications }\end{array}$ \\
\hline $\begin{array}{l}\text { Jordan, et al. } \\
2016^{24}\end{array}$ & $\begin{array}{l}\text { Prospective } \\
\text { cohort }\end{array}$ & $\begin{array}{l}2004-2010 \\
\text { (Norway), } \\
2000-2010 \\
\text { (Wales and } \\
\text { Denmark) }\end{array}$ & $\begin{array}{l}\text { Live births, still } \\
\text { births, fetal deaths } \\
\text { after } 20 \text { weeks ( } 24 \\
\text { weeks in Wales) }\end{array}$ & $\begin{array}{l}\text { One or more prescription for an } \\
\text { antidepressant (Wales) or dispensed } \\
\text { (Norway and Denmark), } 91 \text { days } \\
\text { either side of the first day of the LMP }\end{array}$ & $\begin{array}{l}\text { Did not use any } \\
\text { antidepressant during } \\
\text { the } 91 \text { days either side of } \\
\text { the first day of the LMP }\end{array}$ & n.d. \\
\hline $\begin{array}{l}\text { Furu, et al. } \\
2015^{16}\end{array}$ & $\begin{array}{l}\text { Prospective } \\
\text { cohort }\end{array}$ & $\begin{array}{l}1996-2010 \\
\text { (Denmark, } \\
\text { Finland, Iceland, } \\
\text { Norway and } \\
\text { Sweden) }\end{array}$ & Live births only & $\begin{array}{l}\text { Use of a single, consistent SSRI } \\
\text { between at least one month before } \\
\text { conception and day } 84 \text { of the } \\
\text { pregnancy, identified by redeemed } \\
\text { prescriptions }\end{array}$ & $\begin{array}{l}\text { No redeemed } \\
\text { prescriptions for } \\
\text { antidepressants from } 30 \\
\text { days before the first day } \\
\text { of the LMP to } 97 \text { days } \\
\text { after the LMP }\end{array}$ & $\begin{array}{l}15 \% \text { of SSRI-using and } 1 \% \text { of non-SSRI- } \\
\text { using mothers }\end{array}$ \\
\hline $\begin{array}{l}\text { Berard, et al. } \\
2015^{15}\end{array}$ & $\begin{array}{l}\text { Prospective } \\
\text { cohort }\end{array}$ & $\begin{array}{l}1998-2010 \\
\text { (Canada) }\end{array}$ & Live births only & $\begin{array}{l}\text { Continuous prescription of } \\
\text { antidepressants for at least } 12 \\
\text { months before the LMP and during } \\
\text { pregnancy }\end{array}$ & $\begin{array}{l}\text { Depressed and not using } \\
\text { antidepressant } \\
\text { medications }\end{array}$ & $\begin{array}{l}93 \% \text { of mothers using sertraline } \\
93 \% \text { of mothers using non-sertraline } \\
\text { SSRIs } \\
94 \% \text { of mothers using non-SSRI } \\
\text { antidepressants } \\
84 \% \text { of mothers in the comparison } \\
\text { group }\end{array}$ \\
\hline $\begin{array}{l}\text { Huybrechts, } \\
\text { et al. } 2014^{23}\end{array}$ & $\begin{array}{l}\text { Retrospective } \\
\text { cohort }\end{array}$ & $\begin{array}{l}2000-2007 \\
\text { (United States) }\end{array}$ & Live births only & $\begin{array}{l}\text { Overlap between pharmacy } \\
\text { dispensing of antidepressants and } \\
\text { the first trimester of pregnancy }\end{array}$ & $\begin{array}{l}\text { No dispensing of } \\
\text { antidepressants during } \\
\text { the first trimester of } \\
\text { pregnancy }\end{array}$ & $\begin{array}{l}\text { Among SSRI users: } 4 \% \text { used } \\
\text { antidiabetics, } 16 \% \text { used } \\
\text { anticonvulsants, } 21 \% \text { used } \\
\text { antipsychotics, } 7 \% \text { used anxiolytics, } \\
32 \% \text { used benzodiazepines, } 29 \% \text { used } \\
\text { other hypnotic agents, } 8 \% \text { used } \\
\text { barbiturates and } 8 \% \text { used a suspected } \\
\text { teratogen }\end{array}$ \\
\hline $\begin{array}{l}\text { Ban, et al. } \\
2014^{14}\end{array}$ & $\begin{array}{l}\text { Prospective } \\
\text { cohort }\end{array}$ & $\begin{array}{l}1990-2009 \\
\text { (United Kingdom) }\end{array}$ & Live births only & $\begin{array}{l}\text { Presence of SSRI or TCA prescriptions } \\
\text { in records from four weeks before to } \\
12 \text { weeks after the first day of the } \\
\text { estimated LMP. Dual usage of SSRIs } \\
\text { excluded }\end{array}$ & $\begin{array}{l}\text { Depressed or non- } \\
\text { depressed and not using } \\
\text { antidepressants }\end{array}$ & n.d. \\
\hline $\begin{array}{l}\text { Vasilakis- } \\
\text { Scaramozza, } \\
\text { et al. } 2017^{29}\end{array}$ & $\begin{array}{l}\text { Retrospective } \\
\text { cohort }\end{array}$ & $\begin{array}{l}1991-2002 \\
\text { (United Kingdom) }\end{array}$ & $\begin{array}{l}\text { Live births, stillbirths, } \\
\text { and therapeutic } \\
\text { abortions }>20 \\
\text { weeks' gestation }\end{array}$ & $\begin{array}{l}\text { Prescription receipt for an } \\
\text { antidepressant from } 180 \text { to } 335 \text { days } \\
\text { prior to the delivery date for live } \\
\text { births and from } 70 \text { to } 225 \text { days prior } \\
\text { to the delivery date for stillbirths and } \\
\text { therapeutic abortions }\end{array}$ & $\begin{array}{l}\text { No receipt for an } \\
\text { antidepressant during } \\
\text { pregnancy }\end{array}$ & $\begin{array}{l}\text { Less than } 1 \% \text { of women using an } \\
\text { antidepressant used insulin or a } \\
\text { teratogen during the first trimester of } \\
\text { pregnancy }\end{array}$ \\
\hline $\begin{array}{l}\text { Margulis, et } \\
\text { al. } 2013^{26}\end{array}$ & $\begin{array}{l}\text { Retrospective } \\
\text { cohort }\end{array}$ & $\begin{array}{l}1996-2010 \\
\text { (United Kingdom) }\end{array}$ & Live births only & $\begin{array}{l}\text { Women with one or more } \\
\text { prescriptions for a single SSRI, } \\
\text { overlapping with the first trimester }\end{array}$ & $\begin{array}{l}\text { Women with no } \\
\text { antidepressant } \\
\text { prescriptions in the three }\end{array}$ & $\begin{array}{l}\text { Among SSRI users: } 18 \% \text { had any } \\
\text { prescription for non-SSRI } \\
\text { psychotropics, } 1 \% \text { had any }\end{array}$ \\
\hline
\end{tabular}




\begin{tabular}{|c|c|c|c|c|c|c|}
\hline & & & & of pregnancy & $\begin{array}{l}\text { months before through } \\
\text { to the first or second } \\
\text { trimester of pregnancy }\end{array}$ & $\begin{array}{l}\text { prescriptions for anticonvulsants in } \\
\text { and } 4 \% \text { had non-antidepressant drugs } \\
\text { prescribed in the baseline year }\end{array}$ \\
\hline $\begin{array}{l}\text { Nordeng, et } \\
\text { al. } 2012^{27}\end{array}$ & $\begin{array}{l}\text { Prospective } \\
\text { cohort }\end{array}$ & $\begin{array}{l}1999-2005 \\
\text { (Norway) }\end{array}$ & $\begin{array}{l}\text { Live births, fetal } \\
\text { deaths, or induced } \\
\text { abortions after } \\
\text { gestational week } 12\end{array}$ & $\begin{array}{l}\text { Dispensed prescription for any } \\
\text { antidepressant from } 30 \text { days before } \\
\text { the first day of the LMP until } 97 \text { days } \\
\text { after the LMP }\end{array}$ & $\begin{array}{l}\text { No reported use of any } \\
\text { antidepressants in the six } \\
\text { months before or during } \\
\text { pregnancy }\end{array}$ & n.d. \\
\hline $\begin{array}{l}\text { Diav-Citrin, } \\
\text { et al. } 2008^{33}\end{array}$ & $\begin{array}{l}\text { Prospective } \\
\text { cohort }\end{array}$ & $\begin{array}{l}1994-2002 \\
\text { (Israel and Italy), } \\
2002-2005 \\
\text { (Germany) }\end{array}$ & Live births only & $\begin{array}{l}\text { Dispensed prescription for } \\
\text { paroxetine or fluoxetine from } 30 \\
\text { days before the first day of the LMP } \\
\text { till } 97 \text { days after the LMP }\end{array}$ & $\begin{array}{l}\text { Women who contacted a } \\
\text { centre regarding the use } \\
\text { of medications known } \\
\text { not to be harmful in } \\
\text { similar timeframes }\end{array}$ & n.d. \\
\hline $\begin{array}{l}\text { Oberlander, } \\
\text { et al. } 2008^{28}\end{array}$ & $\begin{array}{l}\text { Prospective } \\
\text { cohort }\end{array}$ & $\begin{array}{l}1998-2001 \\
\text { (Canada) }\end{array}$ & Live births only & $\begin{array}{l}\text { Dispensed antidepressant } \\
\text { prescription from the LMP to } 90 \text { days } \\
\text { after the LMP }\end{array}$ & $\begin{array}{l}\text { No dispensed } \\
\text { prescriptions for } \\
\text { antidepressants or } \\
\text { benzodiazepines }\end{array}$ & $\begin{array}{l}\text { Women taking anticonvulsants were } \\
\text { excluded }\end{array}$ \\
\hline $\begin{array}{l}\text { Davis, et al. } \\
2007^{21}\end{array}$ & $\begin{array}{l}\text { Retrospective } \\
\text { cohort }\end{array}$ & $\begin{array}{l}1996-2000 \\
\text { (United States) }\end{array}$ & Live births only & $\begin{array}{l}\text { Dispensed antidepressant during the } \\
\text { first trimester of pregnancy }\end{array}$ & $\begin{array}{l}\text { Women who were not } \\
\text { prescribed } \\
\text { antidepressants at any } \\
\text { time during pregnancy }\end{array}$ & n.d. \\
\hline $\begin{array}{l}\text { Kulin, et al. } \\
1998^{25}\end{array}$ & $\begin{array}{l}\text { Retrospective } \\
\text { cohort }\end{array}$ & $\begin{array}{l}\text { Unknown time } \\
\text { period (United } \\
\text { States and } \\
\text { Canada) }\end{array}$ & $\begin{array}{l}\text { Live births, } \\
\text { spontaneous } \\
\text { abortions, } \\
\text { therapeutic abortions } \\
\text { and stillbirths }\end{array}$ & $\begin{array}{l}\text { Use of sertraline or fluoxetine during } \\
\text { the first trimester of pregnancy }\end{array}$ & $\begin{array}{l}\text { Use of non-harmful } \\
\text { medications during } \\
\text { pregnancy }\end{array}$ & n.d. \\
\hline $\begin{array}{l}\text { Louik, et al. } \\
2014^{18}\end{array}$ & $\begin{array}{l}\text { Prospective } \\
\text { case-control }\end{array}$ & $\begin{array}{l}1993-2004 \\
\text { (United States) }\end{array}$ & Live births only & $\begin{array}{l}\text { Use of any antidepressant from } 28 \\
\text { days before the LMP to } 112 \text { days } \\
\text { after the LMP }\end{array}$ & $\begin{array}{l}\text { No use of any } \\
\text { antidepressant at any } \\
\text { time from } 56 \text { days prior } \\
\text { to the LMP }\end{array}$ & n.d. \\
\hline $\begin{array}{l}\text { De Jonge, et } \\
\text { al. } 2013^{31}\end{array}$ & $\begin{array}{l}\text { Retrospective } \\
\text { case-control }\end{array}$ & $\begin{array}{l}1998-2008 \\
\text { (Netherlands) }\end{array}$ & $\begin{array}{l}\text { Live births, stillbirths, } \\
\text { spontaneous } \\
\text { abortions and } \\
\text { terminations of } \\
\text { pregnancy }\end{array}$ & $\begin{array}{l}\text { The antidepressant was registered to } \\
\text { be used during the first } 13 \text { weeks } \\
\text { after the first day of the LMP }\end{array}$ & $\begin{array}{l}\text { The antidepressant was } \\
\text { not registered to be used } \\
\text { during the first } 13 \text { weeks } \\
\text { after the first day of the } \\
\text { LMP }\end{array}$ & n.d. \\
\hline $\begin{array}{l}\text { Polen, et al. } \\
2013^{32}\end{array}$ & $\begin{array}{l}\text { Retrospective } \\
\text { case-control }\end{array}$ & $\begin{array}{l}1997-2007 \\
\text { (United States) }\end{array}$ & $\begin{array}{l}\text { Live births, still births } \\
\geq 20 \text { weeks gestation } \\
\text { and elective } \\
\text { terminations }\end{array}$ & $\begin{array}{l}\text { Reported use of venlafaxine from } \\
\text { one month before conception } \\
\text { through to the third month of } \\
\text { pregnancy }\end{array}$ & $\begin{array}{l}\text { No reported use of any } \\
\text { antidepressant from } \\
\text { three months before } \\
\text { conception through the } \\
\text { end of pregnancy }\end{array}$ & n.d. \\
\hline $\begin{array}{l}\text { Alwan, et al. } \\
2010^{30}\end{array}$ & $\begin{array}{l}\text { Retrospective } \\
\text { case-control }\end{array}$ & $\begin{array}{l}1997-2004 \\
\text { (United States) }\end{array}$ & $\begin{array}{l}\text { Live births and fetal } \\
\text { deaths after } 20 \\
\text { weeks gestation }\end{array}$ & $\begin{array}{l}\text { Reported use of bupropion anytime } \\
\text { between one month before and } \\
\text { three months after conception }\end{array}$ & $\begin{array}{l}\text { Did not use any } \\
\text { antidepressant at any } \\
\text { time during pregnancy }\end{array}$ & n.d. \\
\hline
\end{tabular}

LMP, Last menstrual period; n.d., no data; SSRI, Selective serotonin reuptake inhibitor; TCA, tricyclic antidepressant 
Table 2 Systematic review of the individual studies, reporting antidepressant use during the first trimester of pregnancy and odds ratios for congenital heart defects

\begin{tabular}{|c|c|c|c|c|c|c|c|c|c|}
\hline \multirow{2}{*}{$\begin{array}{l}\text { Author, Year } \\
\text { Published }\end{array}$} & \multicolumn{2}{|c|}{ Cohort / Case } & \multicolumn{2}{|c|}{ Comparison } & \multirow{2}{*}{$\begin{array}{l}\text { Antidepressant } \\
\text { Used }\end{array}$} & \multirow{2}{*}{$\begin{array}{l}\text { Crude Odds Ratio } \\
(95 \% \mathrm{Cl})\end{array}$} & \multirow{2}{*}{$\begin{array}{l}\text { Adjusted Odds } \\
\text { Ratio }(95 \% \mathrm{Cl})\end{array}$} & \multirow[t]{2}{*}{ Odds Ratio Adjusted For } & \multirow{2}{*}{$\begin{array}{l}\text { Meta Analysis } \\
\text { That The Data } \\
\text { Was Used In }\end{array}$} \\
\hline & $\begin{array}{l}\mathrm{N} \\
\text { Events }\end{array}$ & N Total & $\begin{array}{l}\mathrm{N} \\
\text { Events }\end{array}$ & N Total & & & & & \\
\hline \multirow[t]{3}{*}{$\begin{array}{l}\text { Jordan, et al. } \\
2016^{24}\end{array}$} & 121 & 12,962 & 4,503 & 506,155 & SSRI or SNRI & $1.03(0.86$ to 1.24$)$ & $1.00(0.82$ to 1.21$)$ & Smoking and socioeconomic status & $\begin{array}{l}\text { Any } \\
\text { antidepressant }\end{array}$ \\
\hline & 101 & 10,959 & 4,047 & 449,643 & SSRI monotherapy & $1.00(0.82$ to 1.22$)$ & \multirow[t]{2}{*}{ n.d. } & \multirow[t]{2}{*}{ n.d. } & SSRI group \\
\hline & 18 & 1,448 & 4,503 & 506,155 & SNRI & $\begin{array}{l}1.40(0.88 \text { to } \\
2.23)^{*}\end{array}$ & & & SNRI group \\
\hline \multirow[t]{3}{*}{$\begin{array}{l}\text { Furu, et al. } \\
2015^{16}\end{array}$} & 530 & 34,535 & \multirow[t]{3}{*}{26,745} & \multirow[t]{3}{*}{$2,266,875$} & $\begin{array}{l}\text { SSRI or } \\
\text { venlafaxine }\end{array}$ & $\begin{array}{l}1.32(1.21 \text { to } \\
1.44)^{*}\end{array}$ & \multirow[t]{2}{*}{ n.d. } & \multirow[t]{2}{*}{ n.d. } & $\begin{array}{l}\text { Any } \\
\text { antidepressant }\end{array}$ \\
\hline & 490 & 31,772 & & & SSRI & $\begin{array}{l}1.33(1.21 \text { to } \\
1.45)^{*}\end{array}$ & & & SSRI group \\
\hline & 40 & 2,763 & & & $\begin{array}{l}\text { Venlafaxine } \\
\text { (monotherapy or } \\
\text { polytherapy) }\end{array}$ & 1.23 (0.90 to 1.68$)$ & $1.14(0.82$ to 1.57$)$ & $\begin{array}{l}\text { Maternal age, year of birth, birth order, } \\
\text { smoking, maternal diabetes, country and use } \\
\text { of other medications }\end{array}$ & SNRI group \\
\hline \multirow[t]{2}{*}{$\begin{array}{l}\text { Berard, et al. } \\
2015^{15}\end{array}$} & 89 & 3,625 & \multirow[t]{2}{*}{344} & \multirow[t]{2}{*}{14,868} & $\begin{array}{l}\text { Any } \\
\text { antidepressant }\end{array}$ & $\begin{array}{l}1.06(0.84 \text { to } \\
1.35)^{*}\end{array}$ & \multirow[t]{2}{*}{ n.d. } & \multirow[t]{2}{*}{ n.d. } & $\begin{array}{l}\text { Any } \\
\text { antidepressant }\end{array}$ \\
\hline & 61 & 2,329 & & & SSRI & $\begin{array}{l}1.14 \text { (0.86 to } \\
1.50)^{*}\end{array}$ & & & SSRI group \\
\hline \multirow[t]{4}{*}{$\begin{array}{l}\text { Huybrechts, } \\
\text { et al. } 2014^{23}\end{array}$} & 580 & 64,389 & \multirow[t]{4}{*}{6,403} & \multirow[t]{4}{*}{885,115} & $\begin{array}{l}\text { Any } \\
\text { antidepressant }\end{array}$ & $\begin{array}{l}1.25(1.15 \text { to } \\
1.36)\end{array}$ & \multirow[t]{4}{*}{ n.d. } & \multirow[t]{4}{*}{ n.d. } & $\begin{array}{l}\text { Any } \\
\text { antidepressant }\end{array}$ \\
\hline & 416 & 46,144 & & & SSRI & $\begin{array}{l}1.25 \text { (1.13 to } \\
1.38)\end{array}$ & & & SSRI group \\
\hline & 75 & 6,904 & & & SNRI & $\begin{array}{l}1.51 \text { (1.20 to } \\
1.89)\end{array}$ & & & SNRI group \\
\hline & 42 & 5,954 & & & TCA & $0.97(0.72$ to 1.32$)$ & & & TCA group \\
\hline \multirow[t]{3}{*}{$\begin{array}{l}\text { Ban, et al. } \\
2014^{14}\end{array}$} & 90 & 10,401 & \multirow[t]{3}{*}{2,556} & \multirow[t]{3}{*}{338,726} & $\begin{array}{l}\text { SSRI or TCA } \\
\text { mono-or } \\
\text { polytherapy }\end{array}$ & $\begin{array}{l}1.15(0.93 \text { to } \\
1.42)^{*}\end{array}$ & \multirow[t]{3}{*}{ n.d. } & \multirow[t]{3}{*}{ n.d. } & $\begin{array}{l}\text { Any } \\
\text { antidepressant }\end{array}$ \\
\hline & 68 & 7,683 & & & SSRI monotherapy & $\begin{array}{l}1.17(0.92 \text { to } \\
1.50)^{*}\end{array}$ & & & SSRI group \\
\hline & 20 & 2,428 & & & TCA monotherapy & $\begin{array}{l}1.09(0.70 \text { to } \\
1.70)^{*}\end{array}$ & & & TCA group \\
\hline \multirow{3}{*}{$\begin{array}{l}\text { Vasilakis- } \\
\text { Scaramozza, } \\
\text { et al. } 2013^{29}\end{array}$} & 18 & 3,287 & \multirow[t]{3}{*}{28} & \multirow[t]{3}{*}{6,617} & SSRI or TCA & $\begin{array}{l}1.30(0.72 \text { to } \\
2.35)^{*}\end{array}$ & n.d. & n.d. & $\begin{array}{l}\text { Any } \\
\text { antidepressant }\end{array}$ \\
\hline & 7 & 1,825 & & & SSRI & $\begin{array}{l}0.91 \text { (0.40 to } \\
2.08)^{*}\end{array}$ & & & SSRI group \\
\hline & 11 & 1,608 & & & TCA & $\begin{array}{l}1.62(0.81 \text { to } \\
3.26)^{*}\end{array}$ & & & TCA group \\
\hline $\begin{array}{l}\text { Margulis, et } \\
\text { al. } 2013^{26}\end{array}$ & 16 & 3,046 & 48 & 8,991 & SSRI & $\begin{array}{l}0.98 \text { (0.56 to. } \\
1.73)^{*}\end{array}$ & 1.09 (0.66 to 1.79$)$ & Logistic regression & $\begin{array}{l}\text { Any } \\
\text { antidepressant }\end{array}$ \\
\hline
\end{tabular}




\begin{tabular}{|c|c|c|c|c|c|c|c|c|c|c|}
\hline & & & & & & & & & and SSRI group & $\beta_{2}^{3}$ \\
\hline \multirow[t]{2}{*}{$\begin{array}{l}\text { Nordeng, et } \\
\text { al. } 2012^{27}\end{array}$} & 6 & 556 & 541 & 61,648 & $\begin{array}{l}\text { Any } \\
\text { antidepressant }\end{array}$ & $\begin{array}{l}1.23(0.55 \text { to } \\
2.77)^{*}\end{array}$ & $1.24(0.55$ to 2.82$)$ & \multirow{2}{*}{$\begin{array}{l}\text { Maternal depression, maternal age at delivery, } \\
\text { parity and use of psychotropic medications } \\
\text { during pregnancy }\end{array}$} & $\begin{array}{l}\text { Any } \\
\text { antidepressant }\end{array}$ & \multirow{2}{*}{ 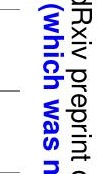 } \\
\hline & 6 & 462 & & & SSRI & $\begin{array}{l}1.49(0.66 \text { to } \\
3.34)^{*}\end{array}$ & 1.51 (0.67 to 3.43$)$ & & SSRI group & \\
\hline $\begin{array}{l}\text { Diav-Citrin, et } \\
\text { al. } 2008^{33}\end{array}$ & 14 & 601 & 8 & 1,359 & $\begin{array}{l}\text { Paroxetine or } \\
\text { fluoxetine }\end{array}$ & $\begin{array}{l}4.03(1.68 \text { to } \\
9.65)^{*}\end{array}$ & n.d. & n.d. & $\begin{array}{l}\text { Any } \\
\text { antidepressant } \\
\text { and SSRI group }\end{array}$ & 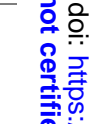 \\
\hline \multirow[t]{3}{*}{$\begin{array}{l}\text { Oberlander, } \\
\text { et al. } 2008^{28}\end{array}$} & 19 & 2,709 & \multirow[t]{3}{*}{512} & \multirow[t]{3}{*}{107,320} & $\begin{array}{l}\text { SSRI or SNRI } \\
\text { monotherapy }\end{array}$ & $\begin{array}{l}1.47(0.93 \text { to } \\
2.33)^{*}\end{array}$ & \multirow[t]{3}{*}{ n.d. } & \multirow[t]{3}{*}{ n.d. } & $\begin{array}{l}\text { Any } \\
\text { antidepressant }\end{array}$ & \multirow{3}{*}{ 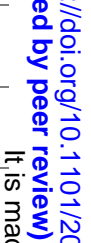 } \\
\hline & 18 & 2,459 & & & SSRI monotherapy & $\begin{array}{l}1.54(0.96 \text { to } \\
2.47)^{*}\end{array}$ & & & SSRI group & \\
\hline & 1 & 250 & & & $\begin{array}{l}\text { Venlafaxine } \\
\text { monotherapy }\end{array}$ & $\begin{array}{l}0.84(0.12 \text { to } \\
5.98)^{*}\end{array}$ & & & SNRI group & \\
\hline \multirow[t]{2}{*}{$\begin{array}{l}\text { Davis, et al. } \\
2007^{21}\end{array}$} & 17 & 805 & 1,171 & 49,031 & SSRI & $\begin{array}{l}0.88(0.54 \text { to } \\
1.43)^{*}\end{array}$ & \multirow[t]{2}{*}{ n.d. } & \multirow[t]{2}{*}{ n.d. } & $\begin{array}{l}\text { Any } \\
\text { antidepressant } \\
\text { and SSRI group }\end{array}$ & \\
\hline & 2 & 167 & 1,186 & 49,669 & TCA & $\begin{array}{l}0.50(0.12 \text { to } \\
2.00)^{*}\end{array}$ & & & $\begin{array}{l}\text { Any } \\
\text { antidepressant } \\
\text { and TCA group }\end{array}$ & \\
\hline $\begin{array}{l}\text { Kulin, et al. } \\
1998^{25}\end{array}$ & 2 & 267 & 4 & 267 & SSRI & $\begin{array}{l}0.50(0.09 \text { to } \\
2.73)^{*}\end{array}$ & n.d. & n.d. & $\begin{array}{l}\text { Any } \\
\text { antidepressant } \\
\text { and SSRI group }\end{array}$ & 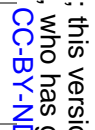 \\
\hline \multirow[t]{3}{*}{$\begin{array}{l}\text { Louik, et al. } \\
2014^{18}\end{array}$} & 447 & 8,805 & 370 & 8,611 & $\begin{array}{l}\text { Any } \\
\text { antidepressant }\end{array}$ & $\begin{array}{l}1.19(1.03 \text { to } \\
1.37)^{*}\end{array}$ & \multirow[t]{3}{*}{ n.d. } & \multirow[t]{3}{*}{ n.d. } & $\begin{array}{l}\text { Any } \\
\text { antidepressant }\end{array}$ & \multirow{3}{*}{ 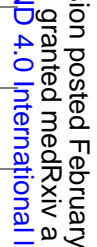 } \\
\hline & 349 & & 290 & & SSRI & $\begin{array}{l}1.18(1.01 \text { to } \\
1.39)^{*}\end{array}$ & & & SSRI group & \\
\hline & 29 & & 32 & & TCA & $\begin{array}{l}0.89(0.54 \text { to } \\
1.47)^{*}\end{array}$ & & & TCA group & \\
\hline $\begin{array}{l}\text { De Jonge, et } \\
\text { al. } 2013^{31}\end{array}$ & 14 & 873 & 281 & 29,223 & SSRI & $\begin{array}{l}1.68(0.98 \text { to } \\
2.88)^{*}\end{array}$ & n.d. & n.d. & $\begin{array}{l}\text { Any } \\
\text { antidepressant } \\
\text { and SSRI group }\end{array}$ & 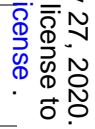 \\
\hline $\begin{array}{l}\text { Polen, et al. } \\
2013^{32}\end{array}$ & 74 & 15,758 & 14 & 8,002 & Venlafaxine & $\begin{array}{l}2.69(1.52 \text { to } \\
4.77)^{*}\end{array}$ & n.d. & n.d. & $\begin{array}{l}\text { Any } \\
\text { antidepressant } \\
\text { and SNRI group }\end{array}$ & 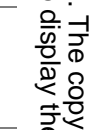 \\
\hline $\begin{array}{l}\text { Alwan, et al. } \\
2010^{30}\end{array}$ & 34 & 6,853 & 26 & 5,869 & Bupropion & $\begin{array}{l}1.12(0.67 \text { to } \\
1.87)^{*}\end{array}$ & $1.4(0.8$ to 2.5$)$ & $\begin{array}{l}\text { Maternal race, obesity, smoking and family } \\
\text { income }\end{array}$ & $\begin{array}{l}\text { Any } \\
\text { antidepressant }\end{array}$ & \\
\hline \multicolumn{10}{|c|}{$\begin{array}{l}\mathrm{Cl} \text {, confidence interval; SSRI, selective serotonin reuptake inhibitor; SNRI, serotonin norepinephrine reuptake inhibitor; n.d., no data; TCA, tricyclic antidepressant } \\
\text { * Odds ratio and } 95 \% \mathrm{Cl} \text { calculated using RevMan } 5.3\end{array}$} & \\
\hline
\end{tabular}


The total pooled odds ratio from the unadjusted meta-analysis for any antidepressant use was 1.22 (95\% Cl: 1.11 to $1.33, p<0.0001$ ) (figure 2). The corresponding number needed to harm is 442 women taking antidepressants during the first trimester of pregnancy.

Statistically significant odds ratios of 1.22 ( $95 \% \mathrm{Cl}: 1.12$ to $1.33, \mathrm{p}<0.00001$ ) and 1.50 ( $95 \% \mathrm{Cl}: 1.19$ to $1.89, \mathrm{p}<0.0006)$ are reported in figure 3 for the SSRI and SNRI subgroups respectively. The corresponding numbers needed to harm are 198 and 447 women taking SSRIs or SNRIs during the first trimester of pregnancy. The TCA subgroup had a non-statistically significant odds ratio of 1.01 ( $95 \% \mathrm{Cl}$ : 0.82 to $1.25, p=0.52$ ) (figure 3 ). Exclusion of the outliers did not change the significance of the results for the overall or class analyses (supplement 5 ).

Analyses of individual antidepressants produced statistically significant odds ratios of 1.53 (95\% Cl: 1.25 to $1.88, p<0.001$ ) for paroxetine, 1.28 (95\% Cl: 1.01 to $1.62, p=0.04$ ) for fluoxetine, 1.28 (95\% Cl: 1.14 to $1.45, p<0.0001$ ) for sertraline and $1.23(95 \% \mathrm{Cl}: 1.01$ to $1.50, p=0.04)$ for bupropion (figure 4$)$. The pooled odds ratios for citalopram, escitalopram and venlafaxine were not significant (figure 4). Removal of the outlying data ${ }^{28}$ in the citalopram analysis resulted in a statistically significant odds ratio of 1.28 (95\% Cl: 1.12 to $1.46, \mathrm{p}=0.0002$ ), reported in supplement 5 .

Analyses of cohort versus case-control studies resulted in odds ratios of 1.19 (95\% Cl: 1.08 to $1.31, \mathrm{p}=$ 0.003 ) and 1.48 (95\% Cl: 1.04 to $2.11, p=0.03$ ), respectively (figure 5). Analyses of prospective versus retrospective studies resulted in odds ratios of 1.21 (1.09 to 1.34, $p=0.0005)$ and 1.24 (95\% Cl: 0.96 to $1.59, p=0.10$ ), respectively (figure 6).

\section{Discussion}

This meta-analysis of 16 studies from 13 countries including 4,564,798 pregnancy outcomes spanning twenty years confirms that using antidepressants during the first trimester of pregnancy is associated with an increased risk of congenital heart defects. Specifically, the odds of women who use any antidepressant in their first trimester of pregnancy having children with congenital heart defects is 1.1 to 1.3 times greater than the unmedicated group, at a $95 \%$ confidence level.

Our results for the usage of any antidepressant ${ }^{13}$, paroxetine, ${ }^{589}$ fluoxetine $^{10}{ }^{11}$, and sertraline $e^{12}$ are comparable to previous meta-analyses, an overview of which is provided in table 3 . These results indicate that both SNRIs and SSRIs increase the odds of congenital heart defects following maternal usage of these antidepressants during the first trimester of pregnancy, the critical period for cardiac development. Additionally, no increased odds for congenital heart defects following the usage of TCAs during the first trimester of pregnancy were found. This is the first meta-analysis to investigate and compare non-SSRI classes of antidepressants. These data are useful for providing insight into classeffects and possible mechanistic similarities for the formation of heart defects, as both SNRIs and SSRIs inhibit serotonin reuptake. ${ }^{34}$ However, limitations still exist in that there is still a lack of data about commonly used antidepressants, as demonstrated from Australian data; statistics for dispensed medications in 2013 identified that $16 \%$ of women of child bearing age who were dispensed psychoanaleptics were dispensed the SNRI desvenlafaxine. ${ }^{36}$ While our study investigated maternal SNRI usage during pregnancy and congenital heart defects, there was limited data on specific SNRIs and none of the studies investigating SNRIs had specific data on desvenlafaxine. ${ }^{16232428} 32$

Venlafaxine and bupropion were the only SNRIs for which there was enough data to include in the individual antidepressant meta-analysis (figure 4), with respective pooled odds ratios of 1.29 ( $95 \% \mathrm{Cl}$ : 0.98 to 1.69 ) and 1.23 (95\% Cl: 1.01 to 1.50$)$. Bupropion is not registered as an antidepressant in 
Australia and is instead used for nicotine dependence and the dose size for this is $150 \mathrm{mg}$. Comparatively, the reported mean dose of bupropion in the USA from the Louik et al. ${ }^{18}$ study ranged from $254 \mathrm{mg}$ in monotherapy to $354 \mathrm{mg}$ in polytherapy.

SSRIs have been the most investigated class of antidepressants in relation to congenital heart defects, probably as a result of the 2005 paroxetine warning. ${ }^{4}$ Significant odds ratios for paroxetine, fluoxetine and sertraline were found (figure 4). Previous meta-analyses have documented a statistically significant relationship between maternal use of paroxetine, ${ }^{589}$ fluoxetine, ${ }^{1011}$ and sertraline $e^{12}$ during the first trimester of pregnancy and congenital heart defects.

The pooled odds ratios for citalopram and escitalopram were not significant (figure 4) and a 2013 metaanalysis also calculated a non-significant pooled odds ratio for maternal citalopram usage and congenital heart defects. ${ }^{8}$ The removal of outlying data in the citalopram analysis resulted in a statistically significant odds ratio of 1.22 (1.03 to 1.45), therefore it is possible that citalopram may increase the likelihood for congenital heart defects (supplement 5 ); further escitalopram had a limited sample size.

The largest previous meta-analysis was carried out by Shen et al. ${ }^{12}$ and investigated the first trimester use of sertraline and congenital heart defects. Their meta-analysis included 12 cohort studies and had a total of 6,468,241 pregnant women, which is larger than the 4,564,798 pregnancy outcomes in this meta-analysis. We used six of the same studies ${ }^{14-16232728}$ however the remaining six from the Shen et al. meta-analysis were excluded for the following reasons: the Colvin et al. $2011^{37}$ study had a comparison group which had potentially used non-SSRI antidepressants; the Merlob et al. $2009^{38}$ study did not meet our inclusion criteria primarily because of self-reported SSRI usage and an unblinded assessment of pregnancy outcomes; the remaining four studies ${ }^{39-42}$ all reported data from the Swedish birth registry and these data therefore overlap with the Furu 2015 study (supplement 4). ${ }^{16}$ Like the Shen et al. ${ }^{12}$ meta-analysis, our results for sertraline use during the first trimester of pregnancy and congenital heart defects are significant, while demonstrating lower heterogeneity, reflected by the $I^{2}$ value (figure 4 , table 3).

The Davis et al. $2007^{21}$ tricyclic antidepressant data and the Kulin et al. $1998^{25}$ study were classified as outliers due to their wide variances. The Diav-Citrin et al. $2008^{33}$ and Polen et al. $2013^{32}$ studies were classified as outliers because they demonstrated markedly higher intervention effect estimates than the overall odds ratio. Removal of these studies did not change the significance of the results but did decrease the heterogeneity (supplement 5 ).

Results were stratified by study type because it has been established that cohort studies are more rigorous than case-control studies as the study design results in less potential for sampling, observation and recall bias. ${ }^{43}$ Both cohort and case-control studies may be affected by confounding factors, including maternal usage of other medications and maternal smoking. The pooled average odds ratios were similar between both study groups (figure 5). Similarly, prospective studies are more rigorous than retrospective studies because these are conducted before pregnancy outcomes are known, resulting in more control over study features and less potential for recall bias. ${ }^{43} 44$ The pooled average odds ratios were significant for the prospective studies and not significant for the retrospective studies (figure 6).

A strength of our study was the inclusion criteria requiring all studies to be of high quality. Therefore, the meta-analyses reported a direct comparison between women who did or did not use antidepressants during the first trimester of pregnancy. One difficulty in generating high quality pharmacoepidemiological data is proper documentation of data in medical records and administrative 
medRxiv preprint doi: https://doi.org/10.1101/2020.02.24.20027532; this version posted February 27, 2020. The copyright holder for this preprint

(which was not certified by peer review) is the author/funder, who has granted medRxiv a license to display the preprint in perpetuity.

It is made available under a CC-BY-ND 4.0 International license .

databases, because there may be missing data, misclassification of medication usage or birth outcomes. One limitation of this meta-analysis is confirming whether the women used the antidepressants. Some studies confirmed this by structured interviews, ${ }_{18} 25273033$ while others only relied on antidepressant dispensings which do not verify consumption. ${ }^{162123242829}$

A strength of this meta-analysis was that it was not limited to studies which only investigate live births. Live birth studies are susceptible to selection bias because congenital heart defects can be detected by scans which generally occur between weeks 18 to 22 of pregnancy. ${ }^{45}$ Pregnancies may be terminated if these defects are detected and these cases or events will be missed if only live births are assessed. Additionally, congenital heart defects may be present in stillbirths. Therefore, restricting analyses to live births may underestimate the relationship between maternal antidepressant use and congenital heart defects. Despite the potential for selection bias, two of the previous meta-analyses restricted pregnancy endpoints to live births only. ${ }^{9}{ }^{12}$ Similar to the results reported here, these meta-analyses found significant results for paroxetine ${ }^{9}$ and sertraline ${ }^{12}$ usage and heart defects, however their odds ratios were slightly larger and have wider confidence intervals (figure 4, table 3).

Our study, limited to high-quality studies, updates the literature on all antidepressants, including antidepressants belonging to the SNRI and TCA classes. There is evidence that maternal use of SNRIs during the first trimester of pregnancy increases the likelihood for congenital heart defects. Additionally, this meta-analysis confirms evidence for the SSRI class having an increased likelihood for causing cardiac defects. Some individual SSRIs, paroxetine, fluoxetine and sertraline, demonstrated statistically significant odds ratios. A statistically significant odds ratio was also demonstrated for the SNRI bupropion. No statistically significant odds ratio was produced for maternal usage of TCAs in the first trimester of pregnancy and congenital heart defects. While this meta-analysis has investigated classes of antidepressants and individual antidepressants in the context of congenital heart defects, further information is needed for some individual antidepressants which are used by women of child-bearing age, such as desvenlafaxine. 


\begin{tabular}{|c|c|c|c|c|c|c|c|c|c|}
\hline $\begin{array}{l}\text { Author, Year } \\
\text { Published }\end{array}$ & $\begin{array}{l}\text { Data Sources } \\
\text { (Search Period) }\end{array}$ & $\begin{array}{l}\text { Study Types } \\
\text { (Setting) }\end{array}$ & $\begin{array}{l}\text { Tool For } \\
\text { Assessing } \\
\text { Study Quality }\end{array}$ & $\begin{array}{l}\mathrm{N} \\
\text { Pregnant } \\
\text { Women }\end{array}$ & $\begin{array}{l}\text { Antidepressants } \\
\text { Investigated }\end{array}$ & $\begin{array}{l}\text { Reported OR/RR } \\
(95 \% \mathrm{CI})\end{array}$ & $\begin{array}{l}I^{2} \text { (p } \\
\text { Value) }\end{array}$ & Study Strengths & Study Weaknesses \\
\hline $\begin{array}{l}\text { Shen, et al. } \\
2017^{12}\end{array}$ & $\begin{array}{l}\text { PubMed and } \\
\text { Web of Science } \\
\text { (inception to } 31 \\
\text { December } \\
\text { 2015) }\end{array}$ & $\begin{array}{l}12 \text { cohort } \\
\text { studies (Europe, } \\
\text { Australia, North } \\
\text { and South } \\
\text { America, Israel) }\end{array}$ & $\begin{array}{l}\text { Newcastle } \\
\text { Ottawa Scale }\end{array}$ & $6,468,241$ & Sertraline & $1.36(1.06$ to 1.74$)$ & $\begin{array}{l}64.4 \% \\
(0.001)\end{array}$ & $\begin{array}{l}\text { Limited to cohort } \\
\text { studies } \\
\text { Modest power size and } \\
\text { population } \\
\text { heterogeneity } \\
\text { explored } \\
\text { One antidepressant } \\
\text { only }\end{array}$ & $\begin{array}{l}\text { Only live births } \\
\text { considered } \\
\text { Case-control } \\
\text { studies not } \\
\text { included } \\
\text { Moderate } \\
\text { heterogeneity } \\
\text { Overlapping study } \\
\text { data } \\
\text { Comparison group } \\
\text { could have used } \\
\text { other } \\
\text { antidepressants }\end{array}$ \\
\hline $\begin{array}{l}\text { Gao, et al. } \\
2017^{10}\end{array}$ & $\begin{array}{l}\text { PubMed and } \\
\text { Web of Science } \\
\text { (inception to } 21 \\
\text { March 2016) }\end{array}$ & $\begin{array}{l}12 \text { cohort } \\
\text { studies (Europe, } \\
\text { North America, } \\
\text { Australia, Israel) }\end{array}$ & $\begin{array}{l}\text { Newcastle } \\
\text { Ottawa Scale }\end{array}$ & $6,450,771$ & Fluoxetine & 1.36 (1.17 to 1.59$)$ & $\begin{array}{l}23.4 \% \\
(0.214)\end{array}$ & $\begin{array}{l}\text { Limited to cohort } \\
\text { studies and population } \\
\text { heterogeneity } \\
\text { explored } \\
\text { Multiple defects } \\
\text { investigated } \\
\text { One antidepressant } \\
\text { only } \\
\text { Live births, fetal deaths } \\
\text { and terminations of } \\
\text { pregnancy included. } \\
\text { Low heterogeneity } \\
\text { observed in results }\end{array}$ & $\begin{array}{l}\text { Case-control } \\
\text { studies not } \\
\text { included }\end{array}$ \\
\hline $\begin{array}{l}\text { Berard, et al. } \\
2016^{5}\end{array}$ & $\begin{array}{l}\text { Embase and } \\
\text { Medline (1996 } \\
\text { to November } \\
\text { 2015) }\end{array}$ & $\begin{array}{l}10 \text { studies, } \\
\text { cohort and } \\
\text { case-control } \\
\text { (North America, } \\
\text { Europe, } \\
\text { Australia) }\end{array}$ & $\begin{array}{l}\text { Inclusion } \\
\text { criteria: } \\
\text { paroxetine } \\
\text { use during T1, } \\
\text { a comparator } \\
\text { group, } \\
\text { information to } \\
\text { calculate an } \\
\text { OR or a given } \\
\text { RR or OR, } \\
\text { investigated }\end{array}$ & $\begin{array}{l}24,303 \\
\text { women } \\
\text { exposed to } \\
\text { paroxetine }\end{array}$ & Paroxetine & 1.23 (1.06 to 1.43$)$ & $\begin{array}{l}1.0 \% \\
(0.436)\end{array}$ & $\begin{array}{l}\text { Limited to case-control } \\
\text { and cohort studies } \\
\text { One antidepressant } \\
\text { only } \\
\text { Low heterogeneity } \\
\text { observed in results } \\
\text { Live births, fetal deaths } \\
\text { and terminations of } \\
\text { pregnancy included. }\end{array}$ & $\begin{array}{l}\text { Comparison group } \\
\text { could have used } \\
\text { other } \\
\text { antidepressants }\end{array}$ \\
\hline
\end{tabular}


congenital

malformations

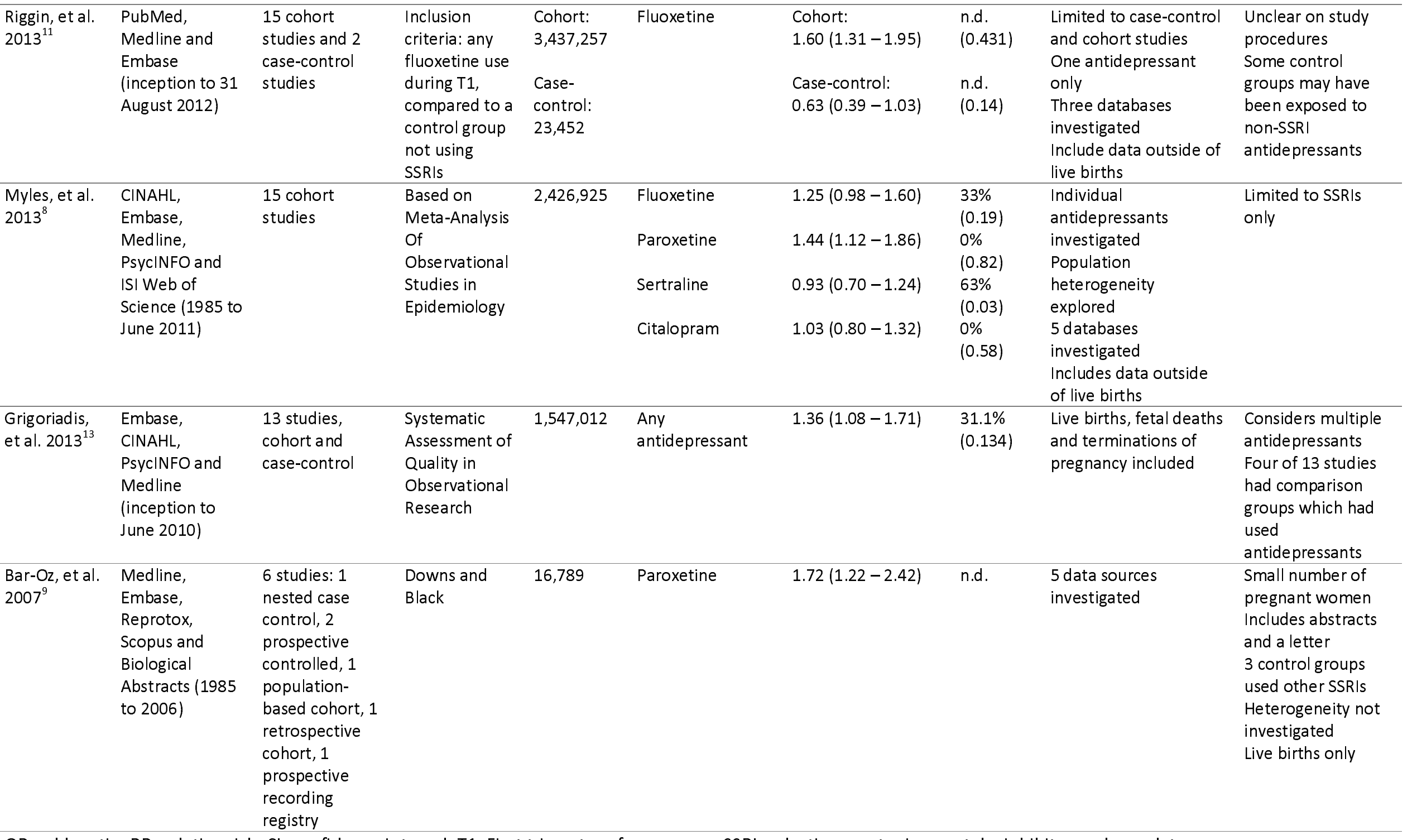

$\mathrm{OR}$, odds ratio; RR, relative risk; $\mathrm{Cl}$, confidence interval; T1, First trimester of pregnancy; SSRI, selective serotonin reuptake inhibitor; n.d., no data 


\section{Meanings Of Study}

This meta-analysis has demonstrated that using antidepressants during the first trimester of pregnancy increases the overall risk for congenital heart defects. TCAs were the only group of antidepressants investigated in this study which did not show an increased odds ratio between maternal use and congenital heart defects. In comparison, SNRIs and SSRIs demonstrated an approximate increased likelihood for congenital heart defects of 1.5 and 1.2 times that of the unmedicated comparison group, respectively.

Analyses of individual antidepressants revealed that the SSRIs paroxetine, fluoxetine and sertraline increased the likelihood of congenital heart defects by 1.5, 1.3 and 1.3 times that of the comparison group respectively. Analyses of individual antidepressants revealed that the SNRI bupropion increased the likelihood of congenital heart defects by 1.2 times that of the comparison group.

Less information is available for some antidepressants such as desvenlafaxine of fluvoxamine, despite desvenlafaxine being the most dispensed psycho-analeptic medication in women of child-bearing age in Australia in $2013 .{ }^{36}$ It is also necessary to investigate risks associated for fluvoxamine because even though it is not as commonly used, medications may become more popular over time or be repurposed.

This meta-analysis has investigated the likelihood of antidepressants causing congenital heart defects however these medications may have other adverse effects which should also be investigated. Therefore, care should be taken when prescribing antidepressant medications to women who are likely to become pregnant, as switching or withdrawing antidepressant therapy is a slow process. Antidepressant therapy can also be ceased prior to conception for women who are well and perhaps more of an emphasis should be placed upon withdrawing medications if they are not needed.

\section{Future Directions}

More pharmacoepidemiological data is needed to build a case for the risk of individual antidepressants causing congenital heart defects, especially those which have not been well studied such as desvenlafaxine and fluvoxamine. Ideally, analyses of all individual antidepressant medications should be completed in the context of other harmful pregnancy outcomes to identify which antidepressants present less risks during pregnancy than others.

If biological targets which play a key role in cardiac formation could be identified, this information could be used to detect medications which may cause congenital heart defects. Firstly, genes involved in cardiac defects can be identified from online sources, such as the Comparative Toxicogenomics Database (http://ctdbase.org/). The most promising genes can be investigated to determine if medications can directly interact with them and molecular modelling may be undertaken, particularly if X-ray crystal structures exist. Medications of interest can be computationally docked into the appropriate binding site to enable potential identification of antidepressants or other medications which may cause congenital heart defects. Laboratory luciferase assays may provide additional evidence for any suspected harmful medications which are identified.

The aim of future work is therefore to identify which medicines may cause congenital heart defects by investigating the mechanisms behind medication-related cardiac malformations.

\section{Conclusions}

Maternal usage of antidepressants during the first trimester of pregnancy increases the risk for congenital heart defects by approximately 1.2 times that of the group not using antidepressants during 
medRxiv preprint doi: https://doi.org/10.1101/2020.02.24.20027532; this version posted February 27, 2020. The copyright holder for this preprint

(which was not certified by peer review) is the author/funder, who has granted medRxiv a license to display the preprint in perpetuity.

It is made available under a CC-BY-ND 4.0 International license.

pregnancy. SNRI and SSRI antidepressants showed an increased risk of 1.5 and 1.2 times respectively, while TCAs were not found to have an increased risk. Individual SSRIs paroxetine, fluoxetine and sertraline have demonstrated an approximate increased likelihood of congenital heart defects by 1.5 , 1.3 and 1.3 times respectively. The SNRI bupropion also demonstrated an approximate 1.2 times increased likelihood of congenital heart defects.

More information is needed about the risks of individual antidepressants, especially for desvenlafaxine, the most dispensed psycho-analeptic in Australia during 2013. The mechanism of antidepressant-related heart defects is currently unknown and future computational and laboratory work could be undertaken to understand these mechanisms and to potentially identify other medications which increase the risk of congenital heart defects. 
medRxiv preprint doi: https://doi.org/10.1101/2020.02.24.20027532; this version posted February 27, 2020. The copyright holder for this preprint

(which was not certified by peer review) is the author/funder, who has granted medRxiv a license to display the preprint in perpetuity.

It is made available under a CC-BY-ND 4.0 International license .

\section{Footnotes}

Contributors: ER and CDV designed the study. CDV did the literature search. CDV and SG extracted data from the identified articles. CDV did the meta-analyses and drafted the manuscript. All authors reviewed the manuscript, contributed to its revision and approved the final version submitted.

Funding: This work was supported by an Australian Government Research Training Program scholarship and NHMRC APP 1110139.

Competing interests: All authors have completed the ICMJE uniform disclosure form at and declare: no support from any organisation for the submitted work; the University of South Australia received payments from the Australian Government Research Training Program, which is a PhD student scholarship, with no financial relationships with any other organisations that might have an interest in the submitted work in the previous three years, excepting no other relationships or activities that could appear to have influenced the submitted work.

License statement: CDV has the right to grant on behalf of all authors and does grant on behalf of all authors, a worldwide licence to the Publishers and its licensees in perpetuity, in all forms, formats and media (whether known now or created in the future), to i) publish, reproduce, distribute, display and store the Contribution, ii) translate the Contribution into other languages, create adaptations, reprints, include within collections and create summaries, extracts and/or, abstracts of the Contribution and convert or allow conversion into any format including without limitation audio, iii) create any other derivative work(s) based in whole or part on the on the Contribution, iv) to exploit all subsidiary rights to exploit all subsidiary rights that currently exist or as may exist in the future in the Contribution, v) the inclusion of electronic links from the Contribution to third party material where-ever it may be located; and, vi) licence any third party to do any or all of the above. All research articles will be made available on an open access basis (with authors being asked to pay an open access fee). The terms of such open access shall be governed by a Creative Commons licence-details as to which Creative Commons licence will apply to the research article are set out in our worldwide licence referred to above.

Ethical approval: Not needed.

Data sharing: Additional data is provided in the supplementary files.

Transparency: The corresponding author affirms that the manuscript is an honest, accurate, and transparent account of the study being reported; that no important aspects of the study have been omitted; and that any discrepancies from the study as planned have been explained.

Patient and Public Involvement Statement: Patients or the public were not involved in the design, or conduct, or reporting, or dissemination plans of our research.

Dissemination Declaration: Dissemination to study participants or patient organisations is not possible/applicable. 
medRxiv preprint doi: https://doi.org/10.1101/2020.02.24.20027532; this version posted February 27, 2020. The copyright holder for this preprint (which was not certified by peer review) is the author/funder, who has granted medRxiv a license to display the preprint in perpetuity. It is made available under a CC-BY-ND 4.0 International license .

\section{References}

1. Jimenez-Solem E, Andersen JT, Petersen M, et al. Prevalence of antidepressant use during pregnancy in Denmark, a nation-wide cohort study. PLOS ONE 2013;8:e63034. doi:10.1371/journal.pone.0063034

2. Mitchell AA, Gilboa SM, Werler MM, Kelley KE, Louik C, Hernandez-Diaz S. Medication use during pregnancy, with particular focus on prescription drugs: 1976-2008. AJOG 2011;205:e1-e8.

3. Hanley GE, Mintzes B. Patterns of psychotropic medicine use in pregnancy in the United States from 2006 to 2011 among women with private insurance. BMC Pregnancy Childbirth 2014;14:242. doi:10.1186/1471-2393-14-242

4. Williams M, Wooltorton E. Paroxetine (Paxil) and congenital malformations. Cmaj 2005;173:1320-1. doi:10.1503/cmaj.051421

5. Berard A, lessa N, Chaabane S, Muanda FT, Boukhris T, Zhao JP. The risk of major cardiac malformations associated with paroxetine use during the first trimester of pregnancy: a systematic review and meta-analysis. $B r$ J Clin Pharmacol 2016;81:589-604. doi:10.1111/bcp.12849

6. Einarson A, Selby P, Koren G. Abrupt discontinuation of psychotropic drugs during pregnancy: fear of teratogenic risk and impact of counselling. J Psychiatry Neurosci 2001;26:44-8.

7. Cohen LS, Altshuler LL, Harlow BL, et al. Relapse of major depression during pregnancy in women who maintain or discontinue antidepressant treatment. Jama 2006;295:499-507. doi:10.1001/jama.295.5.499

8. Myles $\mathrm{N}$, Newall $\mathrm{H}$, Ward $\mathrm{H}$, Large $\mathrm{M}$. Systematic meta-analysis of individual selective serotonin reuptake inhibitor medications and congenital malformations. Aust N Z J Psychiatry 2013;47:1002-12. doi:10.1177/0004867413492219

9. Bar-Oz B, Einarson T, Einarson A, et al. Paroxetine and congenital malformations: meta-analysis and consideration of potential confounding factors. Clin Ther 2007;29:918-26. doi:10.1016/j.clinthera.2007.05.003

10. Gao SY, Wu QJ, Zhang TN, et al. Fluoxetine and congenital malformations: a systematic review and meta-analysis of cohort studies. Br J Clin Pharmacol 2017;83:2134-47. doi:10.1111/bcp.13321

11. Riggin L, Frankel Z, Moretti M, Pupco A, Koren G. The fetal safety of fluoxetine: a systematic review and meta-analysis. JOGC 2013;35:362-9. doi:10.1016/s1701-2163(15)30965-8

12. Shen ZQ, Gao SY, Li SX, et al. Sertraline use in the first trimester and risk of congenital anomalies: a systemic review and meta-analysis of cohort studies. Br J Clin Pharmacol 2017;83:909-22. doi:10.1111/bcp.13161

13. Grigoriadis S, VonderPorten EH, Mamisashvili L, et al. Antidepressant exposure during pregnancy and congenital malformations: is there an association? A systematic review and meta-analysis of the best evidence. J Clin Psychiatry 2013;74:e293-308. doi:10.4088/JCP.12r07966

14. Ban L, Gibson JE, West J, et al. Maternal depression, antidepressant prescriptions, and congenital anomaly risk in offspring: a population-based cohort study. Bjog 2014;121:1471-81. doi:10.1111/1471-0528.12682

15. Berard A, Zhao JP, Sheehy O. Sertraline use during pregnancy and the risk of major malformations. Am J Obstet Gynecol 2015;212:795.e1-.e12. doi:10.1016/j.ajog.2015.01.034

16. Furu K, Kieler $\mathrm{H}$, Haglund $\mathrm{B}$, et al. Selective serotonin reuptake inhibitors and venlafaxine in early pregnancy and risk of birth defects: population based cohort study and sibling design. Bmj 2015;350:h1798. doi:10.1136/bmj.h1798

17. Knudsen TM, Hansen AV, Garne E, Andersen AMN. Increased risk of severe congenital heart defects in offspring exposed to selective serotonin-reuptake inhibitors in early pregnancy - an epidemiological study using validated EUROCAT data. BMC Pregnancy Childbirth 2014;14:

18. Louik C, Kerr S, Mitchell AA. First-trimester exposure to bupropion and risk of cardiac malformations. Pharmacoepidemiol Drug Saf 2014;23:1066-75. doi:10.1002/pds.3661

19. Hartling L, Milne A, Hamm M, et al. Testing the Newcastle Ottawa Scale showed low reliability between individual reviewers. J Clin Epidemiol 2013;66:982-3. doi:10.1016/j.jclinepi.2013.03.003 
20. Lo C, Mertz D, Loeb M. Newcastle-Ottawa Scale: comparing reviewers' to authors' assessments. BMC Med Res Methodol 2014;14:45. doi:10.1186/1471-2288-14-45.

21. Davis RL, Rubanowice D, McPhillips H, et al. Risks of congenital malformations and perinatal events among infants exposed to antidepressant medications during pregnancy. Pharmacoepidemiol Drug Saf 2007;16:1086-94. doi:10.1002/pds.1462

22. Review Manager (RevMan) [program]. 5.4 version. Copenhagen: The Cochrane Collaboration, 2014.

23. Huybrechts KF, Palmsten K, Avorn J, et al. Antidepressant use in pregnancy and the risk of cardiac defects. N Engl J Med 2014;370:2397-407. doi:10.1056/NEJMoa1312828

24. Jordan S, Morris JK, Davies GI, et al. Selective Serotonin Reuptake Inhibitor (SSRI) Antidepressants in Pregnancy and Congenital Anomalies: Analysis of Linked Databases in Wales, Norway and Funen, Denmark. PloS One 2016;11:e0165122. doi:10.1371/journal.pone.0165122

25. Kulin NA, Pastuszak A, Sage SR, et al. Pregnancy outcome following maternal use of the new selective serotonin reuptake inhibitors: a prospective controlled multicenter study. Jama 1998;279:609-10. doi:10.1001/jama.279.8.609

26. Margulis AV, Abou-Ali A, Strazzeri MM, et al. Use of selective serotonin reuptake inhibitors in pregnancy and cardiac malformations: a propensity-score matched cohort in CPRD. Pharmacoepidemiol Drug Saf 2013;22:942-51. doi:10.1002/pds.3462

27. Nordeng H, Van Gelder MMHJ, Spigset O, Koren G, Einarson A, Eberhard-Gran M. Pregnancy outcome after exposure to antidepressants and the role of maternal depression: Results from the Norwegian mother and child cohort study. J Clin Psychopharmacol 2012;32:186-94.

28. Oberlander TF, Warburton W, Misri S, Riggs W, Aghajanian J, Hertzman C. Major congenital malformations following prenatal exposure to serotonin reuptake inhibitors and benzodiazepines using population-based health data. Birth Defects Res B Dev Reprod Toxicol 2008;83:68-76. doi:10.1002/bdrb.20144

29. Vasilakis-Scaramozza C, Aschengrau A, Cabral H, Jick SS. Antidepressant use during early pregnancy and the risk of congenital anomalies. Pharmacotherapy 2013;33:693-700. doi:10.1002/phar.1211

30. Alwan S, Reefhuis J, Botto LD, Rasmussen SA, Correa A, Friedman JM. Maternal use of bupropion and risk for congenital heart defects. AJOG 2010;203:52.e1-6. doi:10.1016/j.ajog.2010.02.015

31. De Jonge L, Zetstra-Van Der Woude PA, Bos HJ, De Jong-Van Den Berg LTW, Bakker MK. Identifying associations between maternal medication use and birth defects using a case-population approach: An exploratory study on signal detection. Drug Saf 2013;36:1069-78. doi:10.1007/s40264-013-0082-2.

32. Polen KND, Rasmussen SA, Riehle-Colarusso T, Reefhuis J. Association between reported venlafaxine use in early pregnancy and birth defects, national birth defects prevention study, 1997-2007. Birth Defects Res A Clin Mol Teratol 2013;97:28-35.

33. Diav-Citrin O, Shechtman S, Weinbaum D, et al. Paroxetine and fluoxetine in pregnancy: a prospective, multicentre, controlled, observational study. Br J Clin Pharmacol 2008;66:695-705. doi:10.1111/j.1365-2125.2008.03261.x

34. Lambert O, Bourin M. SNRIs: mechanism of action and clinical features. Expert Review of Neurotherapeutics 2002;2:849-58. doi:10.1586/14737175.2.6.849

35. Sangkuhl K, Klein TE, Altman RB. Selective serotonin reuptake inhibitors pathway. Pharmacogenetics and genomics 2009;19:907-9. doi:10.1097/FPC.0b013e32833132cb

36. Gadzhanova S, Roughead E. Use of prescription medicines in Australian women of child-bearing age. BMC Pharmacology and Toxicology 2015;16:doi:10.1186/s40360-015-0033-x

37. Colvin L, Slack-Smith L, Stanley FJ, Bower C. Dispensing patterns and pregnancy outcomes for women dispensed selective serotonin reuptake inhibitors in pregnancy. Birth Defects Research Part A Clinical and Molecular Teratology 2011;91:142-52. doi:10.1002/bdra.20773

38. Merlob P, Birk E, Sirota L, et al. Are selective serotonin reuptake inhibitors cardiac teratogens? Echocardiographic screening of newborns with persistent heart murmur. Birth Defects Res A Clin Mol Teratol 2009;85:837-41. doi:10.1002/bdra.20615 
medRxiv preprint doi: https://doi.org/10.1101/2020.02.24.20027532; this version posted February 27, 2020. The copyright holder for this preprint

(which was not certified by peer review) is the author/funder, who has granted medRxiv a license to display the preprint in perpetuity.

It is made available under a CC-BY-ND 4.0 International license.

39. Kornum JB, Nielsen RB, Pedersen L, Mortensen PB, Norgaard M. Use of selective serotonin-reuptake inhibitors during early pregnancy and risk of congenital malformations: Updated analysis. Clinical Epidemiology 2010;2:29-36.

40. Kallen BA, Otterblad Olausson P. Maternal use of selective serotonin re-uptake inhibitors in early pregnancy and infant congenital malformations. Birth Defects Res A Clin Mol Teratol 2007;79:301-8. doi:10.1002/bdra.20327

41. Jimenez-Solem E, Andersen JT, Petersen $M$, et al. Exposure to selective serotonin reuptake inhibitors and the risk of congenital malformations: A nationwide cohort study. BMJ Open 2012;2:

42. Malm H, Artama M, Gissler M, Ritvanen A. Selective serotonin reuptake inhibitors and risk for major congenital anomalies. Obstet Gynecol 2011;118:111-20. doi:10.1097/AOG.0b013e318220edcc

43. Mann CJ. Observational research methods. research design II: cohort, cross sectional, and casecontrol studies. EMJ 2003;20:54. doi:10.1136/emj.20.1.54

44. Norvell DC. Study types and bias - don't judge a study by the abstract's conclusion alone. Evid Based Spine Care J 2010;1:7-10. doi:10.1055/s-0028-1100908

45. Zhang Y-F, Zeng X-L, Zhao E-F, Lu H-W. Diagnostic value of fetal echocardiography for congenital heart disease: a systematic review and meta-analysis. Medicine 2015;94:e1759. doi:10.1097/MD.0000000000001759 


\section{Electronic databases $(\mathrm{n}=2973)$ : \\ PubMed $(n=809) \quad$ Embase $(n=2164)$}

$\rightarrow$ Duplicates removed $(n=287)$

Title or abstract reviews $(\mathrm{n}=2686)$

$\rightarrow$ Failed title or abstract reviews $(n=2601)$

Full-text review $(n=85)$

Excluded ( $n=69)$ :

Not a cohort or case-control study $(n=25)$

Overlapping study $(n=12)$

No cardiac defect outcomes measured $(n=12)$

No antidepressant usage $(n=7)$

Comparison group is unsuitable $(n=7)$

Failed study inclusion criteria $(n=4)$

Not first trimester antidepressant use $(n=2)$

Included in meta-analysis $(n=16)$ :

Cohort studies $(n=12)$

Case-control studies $(n=4)$ 


\section{Cohort / Case Comparison}

Study or Subgroup

Jordan 2016

Berard 2015

Furu 2015

Ban 2014

Louik 2014

Huybrechts 2014

Polen 2013

Vasilakis-Scaramozza 2013

De Jonge 2013

Margulis 2013

Nordeng 2012

Alwan 2010

Oberlander 2008

Diav-Citrin 2008

Davis 2007a

Davis 2007b

Kulin 1998

Total $(95 \% \mathrm{Cl})$

Heterogeneity $\mathrm{Tau}^{2}=0.01 ; \mathrm{Chi}^{2}=28.33, \mathrm{df}=1$

Test for overall effect: $Z=4.32(P<0.0001)$

$14 \quad 873$
Events Total

$4503 \quad 506155$

$344 \quad 14868$

$26745 \quad 2266875$

$2556 \quad 338726$

$370 \quad 8611$

$6403 \quad 885115$

$14 \quad 8002$

$28 \quad 6617$

$281 \quad 29223$

$48 \quad 8991$

$541 \quad 61648$

$26 \quad 5869$

512107320

$8 \quad 1359$

$1171 \quad 49031$

$1186 \quad 49669$

$4 \quad 267$

$\mathbf{4 4 7 4 0 \quad 4 3 4 8 3 4 6}$

$6(P=0.03) ;\left.\right|^{2}=44 \%$
Weight $\quad(95 \% \mathrm{Cl})$

12112962

$89 \quad 3625$

$530 \quad 34535$

$90 \quad 10401$

$447 \quad 8805$

$580 \quad 64389$

$\begin{array}{ll}74 & 15758\end{array}$

$18 \quad 3287$

$16 \quad 3046$

$6 \quad 556$

$34 \quad 6853$

$19 \quad 2709$

$14 \quad 601$

$17 \quad 805$

2167

$2 \quad 267$
$11.4 \% 1.05[0.88,1.26]$

$8.6 \% \quad 1.06[0.84,1.35]$

$17.7 \% \quad 1.32[1.21,1.44]$

$9.8 \% \quad 1.15[0.93,1.42]$

$17.8 \% \quad 1.25[1.15,1.36]$

$2.2 \% \quad 2.69[1.52,4.77]$

$2.1 \% \quad 1.30[0.72,2.35]$

$2.4 \% 1.68[0.98,2.88]$

$2.2 \% \quad 0.98[0.56,1.73]$

$1.2 \% \quad 1.23[0.55,2.77]$

$2.7 \% \quad 1.12[0.67,1.87]$

$3.2 \% \quad 1.47[0.93,2.33]$

$1.0 \% \quad 4.03[1.68,9.65]$

$3.0 \% \quad 0.88[0.54,1.43]$

$0.4 \% \quad 0.50[0.12,2.00]$

$0.3 \% \quad 0.50[0.09,2.73]$
$13.9 \% \quad 1.19[1.03,1.37]$

Odds Ratio

Mantel-Haenszel, Random, 95\% Cl

$100.0 \% \quad 1.22[1.11,1.33]$

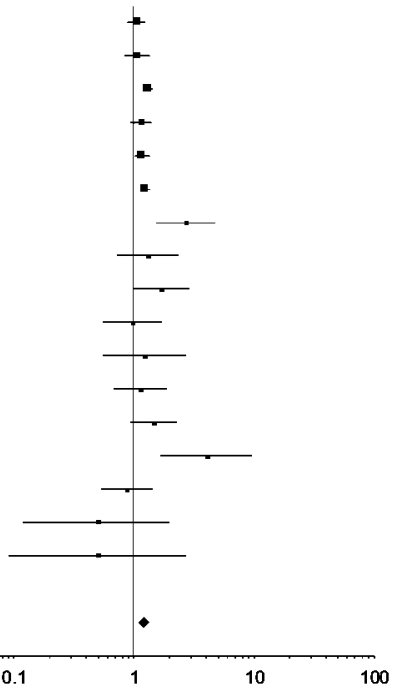




\section{Cohort / Case Comparison}

Study or Subgroup

Events Total

Events Tota

Weight

$(95 \% \mathrm{Cl})$

SSR

Jordan 2016

Berard 2015

$\begin{array}{rr}101 & 10959 \\ 61 & 2329\end{array}$

$4047 \quad 449643$

$344 \quad 14868$

$490 \quad 31772$

267452266875

$68 \quad 7683$

$2556 \quad 338726$

$349 \quad 8805$

$290 \quad 8611$

$416 \quad 46144$

$6403 \quad 885115$

$7 \quad 1825$

$28 \quad 6617$

$281 \quad 29223$

$48 \quad 8991$

$541 \quad 61648$

512107320

$18 \quad 2459$

$14 \quad 601$

$17 \quad 805$

$2 \quad 267$
8.5\% $1.02[0.84,1.25]$

$5.8 \% 1.14[0.86,1.50]$

$14.0 \% \quad 1.31[1.20,1.44]$

$6.9 \% \quad 1.17[0.92,1.50]$

$10.4 \% \quad 1.18[1.01,1.39]$

$13.5 \% \quad 1.25[1.13,1.38]$

$0.9 \% \quad 0.91[0.40,2.08]$

$2.0 \% \quad 1.68[0.98,2.88]$

$1.9 \% \quad 0.98[0.56,1.73]$

$1.0 \% \quad 1.49[0.66,3.34]$

$2.6 \% \quad 1.54[0.96,2.47]$

$0.8 \% 4.03[1.68,9.65]$

$2.5 \% \quad 0.88[0.54,1.43]$

$0.2 \% \quad 0.50[0.09,2.73]$

$71.1 \% \quad 1.22[1.12,1.33]$

\section{Subtotal $(95 \% \mathrm{Cl})$}

1579118030

$42978 \mathbf{4 2 2 8 2 9 4}$

Heterogeneity. Tau $^{2}=0.01 ; \mathrm{Chi}^{2}=19.41, \mathrm{df}=13(\mathrm{P}=0.11) ; \mathrm{I}^{2}=33 \%$

Test for overall effect: $Z=4.44(P<0.00001)$

\section{SNR}

\section{Jordan 2016}

Furu 2015

Huybrechts 2014

Polen 2013

Oberlander 2008

\section{Subtotal $(95 \% \mathrm{Cl})$}

Heterogeneity: $\mathrm{Tau}^{2}=0.02 ; \mathrm{Chi}^{2}=6.01, \mathrm{df}=4(\mathrm{P}=0.20) ; \mathrm{I}^{2}=33 \%$

Test for overall effect: $Z=3.45(P=0.0006)$

TCA

Ban 2014

Louik 2013

Huybrechts 2014

Vasilakis-Scaramozza 2013

Davis 2007b

Subtotal (95\% C)
4503506155

267452266875

6403885115

148002

$512 \quad 107320$

$2.6 \% \quad 1.40[0.88,2.23]$

$4.9 \% \quad 1.23[0.90,1.68]$

$7.3 \% \quad 1.51[1.20,1.89]$

$1.9 \% \quad 2.69[1.52,4.77]$

$0.2 \% \quad 0.84[0.12,5.98]$

$17.1 \% \quad 1.50[1.19,1.89]$

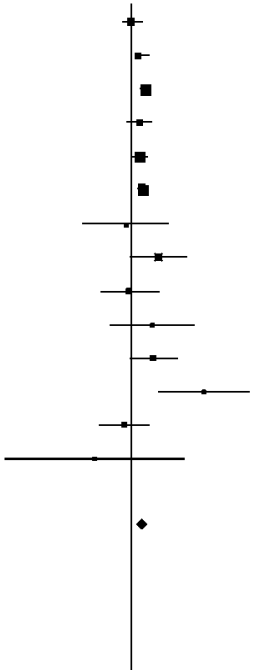

Heterogeneity Tau $^{2}=0.00 ; \mathrm{Chi}^{2}=3.20, \mathrm{df}=4(\mathrm{P}=0.52) ; \mathrm{I}^{2}=0 \%$

Test for overall effect: $Z=0.14(P=0.89)$

$100.0 \% \quad 1.23[1.13,1.34]$

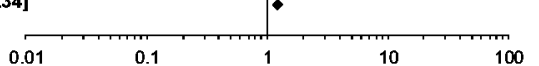




\section{Study or Subgroup}

Cohort / Case

$\begin{array}{lrrrrrrr}\text { Paroxetine } & & & & & & & \\ \text { Jordan 2016 } & 17 & 1069 & 4047 & 449643 & 2.2 \% & 1.78[1.10,2.88] & - \\ \text { Furu 2015 } & 53 & 2879 & 26745 & 2266875 & 5.0 \% & 1.57[1.20,2.06] & - \\ \text { Ban 2014 } & 17 & 1200 & 2556 & 338726 & 2.2 \% & 1.89[1.17,3.06] & -\end{array}$

$\begin{array}{llllll}17 & 1200 & 2556 & 338726 & 2.2 \% & 1.89[1.17,3.00\end{array}$

Huybrechts 201

De Jonge 2013

Nordeng 2012

Oberlander 2008

Diav-Citrin 2008

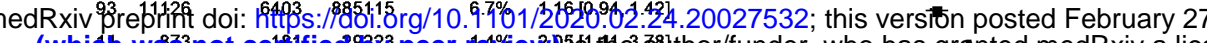

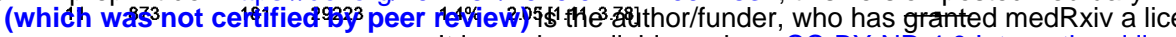
Davis 2007

$\begin{array}{rrrr}1 & 76 & 541 & 61648 \\ 7 & 993 & 512 & 107320 \\ 7 & 348 & 8 & 1359 \\ 4 & 182 & 1184 & 49654\end{array}$

triss mead甲o avadale under a CG-BY ND-4.0-International licer

Subtotal (95\%Cl)

$210 \quad 18746 \quad 42177 \quad 4189563$

$1.0 \% \quad 1.48[0.70,3.13]$

$0.6 \% \quad 3.47[1.25,9.63]$

$0.6 \% \quad 0.92[0.34,2.48]$

Heterogeneity. Tau $^{2}=0.03 ; \mathrm{Chi}^{2}=11.70, \mathrm{df}=8(\mathrm{P}=0.17) ; \mathrm{I}^{2}=32 \%$

Test for overall effect: $Z=4.17(P<0.0001)$

$\begin{array}{lrrrr}\text { Huoxetine } & & & & \\ \text { Jordan 2016 } & 21 & 2601 & 4047 & 449643 \\ \text { Furu 2015 } & 106 & 6250 & 26745 & 2266875 \\ \text { Ban 2014 } & 21 & 3189 & 2556 & 338726 \\ \text { Huybrechts 2014 } & 99 & 11048 & 6403 & 885115 \\ \text { De Jonge 2013 } & 3 & 873 & 65 & 29223 \\ \text { Nordeng 2012 } & 1 & 51 & 541 & 61648 \\ \text { Oberlander 2008 } & 5 & 638 & 512 & 107320 \\ \text { Diav-Citrin 2008 } & 7 & 253 & 8 & 1359\end{array}$

$19.8 \% \quad 1.53[1.25,1.88]$

Subtotal $(95 \% \mathrm{Cl})$

263
$24903 \quad 40877 \quad 4139909$

Heterogeneity. Tau $^{2}=0.04 ; \mathrm{Chi}^{2}=14.53, \mathrm{df}=7(\mathrm{P}=0.04) ; \mathrm{I}^{2}=52 \%$

Test for overall effect: $Z=2.04(P=0.04)$

Sertraline

Jordan 2016

Berard 2015

Furu 2015

Ban 2014

Huybrechts 2014

Nordeng 2012

Oberlander 2008

$\begin{array}{rrrr}17 & 1374 & 4047 & 449643 \\ 10 & 366 & 344 & 14868\end{array}$

$2.2 \% \quad 1.38[0.85,2.23]$

$1.3 \% \quad 1.19[0.63,2.24]$

$7.2 \% \quad 1.28[1.06,1.55]$

$1.3 \% \quad 1.58[0.82,3.06]$

$7.7 \% \quad 1.27[1.07,1.52]$

$0.2 \% \quad 1.15[0.16,8.28]$

$0.5 \% \quad 1.03[0.33,3.23]$

Subtotal (95\% Cl)

$26745 \quad 2266875$

$\begin{array}{llll}9 & 757 & 2556 & 338726\end{array}$

$\begin{array}{llll}129 & 14040 & 6403 & 885115\end{array}$

$\begin{array}{lll}1 & 541 & 61648\end{array}$

$.6 \% \quad 0.90[0.58,1.38]$

$2.6 \% \quad 0.87[0.57,1.34]$

$6.9 \% \quad 1.24[1.02,1.51]$

$1.55[0.49,4.93]$

$0.7 \% \quad 1.65[0.68,3.99]$

$0.6 \% \quad 4.81[1.73,13.37]$

$21.2 \% \quad 1.28[1.01,1.62]$

Heneity $\mathrm{Tau}^{2}=0.00 ; \mathrm{Chi}^{2}=0.69, \mathrm{df}=6(\mathrm{P}=0.99) ; \mathbf{l}^{2}=0 \%$

Test for overall effect $Z=4.12(P<0.0001)$

Citalopram

Jordan 2016

Furu 2015

Ban 2014

De Jonge 2013

Oberlander 2008

$\begin{array}{llll}40 & 4028 & 4047 & 449643\end{array}$

$\begin{array}{lllll}176 & 11193 & 26745 & 2266875\end{array}$

$\begin{array}{llll}17 & 1946 & 2556 & 338726\end{array}$

$\begin{array}{llll}0 & 873 & 35 & 29223\end{array}$

$\begin{array}{llll}3 & 101 & 512 & 107320\end{array}$

$20.4 \% \quad 1.28[1.14,1.45]$

Subtotal $(\mathbf{9 5} \% \mathrm{Cl})$

$\begin{array}{llll}236 & 18141 \quad 33895 & 3191787\end{array}$

Heterogeneity: Tau $^{2}=0.06 ; \mathrm{Chi}^{2}=9.29, \mathrm{df}=4(\mathrm{P}=0.05) ; \mathrm{I}^{2}=57 \%$

Test for overall effect: $Z=1.82(P=0.07)$

\section{Escitalopram}

Jordan 2016

Furu 2015

$\begin{array}{llll}21 & 3236 & 4047 & 449643\end{array}$

$\begin{array}{llll}46 & 3950 & 26745 & 2266875\end{array}$

Ban 2014

$\begin{array}{llll}3 & 333 & 2556 & 338726\end{array}$

Subtotal $(95 \% \mathrm{Cl})$

$\begin{array}{llll}70 & 7519 & 33348 & 3055244\end{array}$

Heterogeneity: Tau $^{2}=0.00 ;$ Chi $^{2}=1.68, \mathrm{df}=2(P=0.43) ; \mathrm{I}^{2}=0 \%$

Test for overall effect: $Z=0.83(P=\mathbf{0 . 4 1})$

Ruvoxamin

Furu 201

Oberlander 2008

$\begin{array}{llll}0 & 28 & 4047 & 449643\end{array}$

$\begin{array}{llll}0 & 255 & 26745 & 2266875\end{array}$

$\begin{array}{llll}0 & 119 & 512 \quad 107320\end{array}$

Subtotal (95\% C)

$\begin{array}{llll}0 & 402 & 31304 & 2823838\end{array}$

Heterogeneity. Tau $^{2}=0.00 ; \mathrm{Chi}^{2}=1.97, \mathrm{df}=2(\mathrm{P}=0.37) ; \mathbf{1}^{2}=0 \%$

Test for overall effect: $Z=0.53(P=0.60)$

Venlafaxin

Furu 201

Oberlander 2008

Polen 2013

$40 \quad 2763$

$26745 \quad 2266875$

$12 \quad 107320$

$\begin{array}{llll}74 & 15758 & 12 & 4038\end{array}$

$4.2 \% \quad 1.10[0.81,1.51]$

$8.6 \% \quad 1.34[1.15,1.55]$

$2.2 \% \quad 1.16[0.72,1.87]$

$0.1 \% \quad 0.47[0.03,7.68]$

$0.4 \% \quad 6.39[2.02,20.21]$

$15.5 \% \quad 1.33[0.98,1.81]$

$.6 \% \quad 0.72[0.47,1.11]$

$4.6 \% \quad 0.99[0.74,1.32]$

$0.5 \% \quad 1.20[0.38,3.73]$

$7.7 \% \quad 0.90[0.71,1.15]$

$0.1 \% \quad 1.93[0.12,31.64]$

$0.1 \% \quad 0.16[0.01,2.63]$

$0.1 \% \quad 0.87[0.05,14.04]$

$0.2 \% \quad 0.65[0.13,3.23]$

$4.2 \% \quad 1.23[0.90,1.68]$

$0.2 \% \quad 0.84[0.12,5.98]$

$1.4 \% \quad 1.58[0.86,2.92]$

$5.8 \% \quad 1.29[0.98,1.69]$

$\begin{array}{llll}115 & 18771 \quad 27269 & 2378233\end{array}$

Subtotal $(95 \% \mathrm{Cl})$

Heterogeneity. Tau $^{2}=0.00 ; \mathrm{Chi}^{2}=0.71, \mathrm{df}=2(\mathrm{P}=0.70) ; \mathrm{I}^{2}=0 \%$

Test for overall effect: $Z=1.79(P=0.07)$

Bupropior

Louik 20

Huybrechts 2014

$19 \quad 4676$

$76 \quad 8856$

$19 \quad 8611$

$6403 \quad 885115$

$\begin{array}{llll}34 & 6853 & 26 & \mathbf{5 8 6 9}\end{array}$

$1.3 \% \quad 1.84[0.98,3.49]$

$6.1 \% \quad 1.19[0.95,1.49]$

$2.0 \% \quad 1.12[0.67,1.87]$

$9.4 \% \quad 1.23[1.01,1.50]$

$\begin{array}{llll}129 & 20385 & 6448 & 899595\end{array}$

$\begin{array}{lccc}\text { Subtotal }(95 \% \mathrm{Cl}) & 129 & 20385 & \mathbf{6 4 4 8} \\ \text { Heterogeneity. } \text { Tau }^{2}=0.00 ; \mathrm{Ch}^{2}=1.77, & \mathrm{df}=2(\mathrm{P}=0.41) ; \mathrm{i}^{2}=0 \%\end{array}$

Test for overall effect: $Z=2.04(P=0.04)$

Total (95\% Cl)

$1310133356 \quad 25646624802364$

$100.0 \% \quad 1.28[1.19,1.39]$
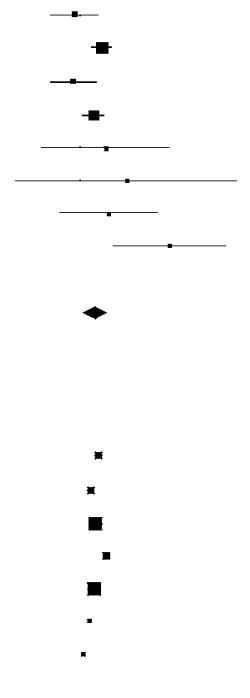

- 


\section{Cohort / Case Comparison}

Events

Total

Weight

Cohort

Jordan 2016

Berard 2015

Furu 2015

Ban 2014

Huybrechts 2014

Vasilakis-Scaramozza 2013

$\begin{array}{llllll}121 & 12962 & 4503 & 506155 & 11.4 \% & 1.05[0.88,1.26]\end{array}$

$\begin{array}{llllll}89 & 3625 & 344 & 14868 & 8.6 \% & 1.06[0.84,1.35]\end{array}$

$\begin{array}{llllll}530 & 34535 & 26745 & 2266785 & 17.7 \% & 1.31[1.20,1.42]\end{array}$

$\begin{array}{llllll}90 & 10401 & 2556 & 338726 & 9.8 \% & 1.15[0.93,1.42]\end{array}$

$\begin{array}{llllll}580 & 64389 & 6403 & 885115 & 17.8 \% & 1.25[1.15,1.36]\end{array}$

$\begin{array}{llllll}18 & 3287 & 28 & 6617 & 2.1 \% & 1.30[0.72,2.35]\end{array}$

$\begin{array}{llllll}16 & 3046 & 48 & 8991 & 2.2 \% & 0.98\end{array}[0.56,1.73]$

$\begin{array}{llllll}6 & 556 & 541 & 61648 & 1.2 \% & 1.23[0.55,2.77]\end{array}$

$\begin{array}{llllll}19 & 2709 & 512 & 107320 & 3.2 \% & 1.47\end{array}[0.93,2.33]$

$\begin{array}{llllll}14 & 601 & 8 & 1359 & 1.0 \% & 4.03[1.68,9.65]\end{array}$

$\begin{array}{llllll}17 & 805 & 1171 & 49031 & 3.0 \% & 0.88\end{array}[0.54,1.43]$

$\begin{array}{llllll}2 & 167 & 1186 & 49669 & 0.4 \% & 0.50[0.12,2.00]\end{array}$

$2 \quad 267$

267

$0.3 \% \quad 0.50[0.09,2.73]$

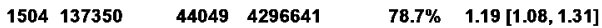

Subtotal (95\% C)

$=0.08) ;\left.\right|^{2}=39 \%$

Heterogeneity $\mathrm{Tau}^{2}=0.01 ; \mathrm{Chi}^{2}=19.58, \mathrm{df}=$

Test for overall effect: $Z=3.64(P=0.0003)$

\section{Case-Control}

Louik 2014

Polen 2013

De Jonge 2013

Awan 2010

$\begin{array}{rr}447 & 8805 \\ 74 & 15758 \\ 14 & 873 \\ 34 & 6853\end{array}$

$\begin{array}{rr}370 & 8611 \\ 14 & 8002 \\ 281 & 29223 \\ 26 & 5869\end{array}$

56932289

$691 \quad 51705$

Subtotal (95\% C)

$=8.68, d f=3(P=0.03) ; 1^{2}=65 \%$

Heterogeneity. Tau $^{2}=0.08 ; \mathrm{Chi}^{2}=8.68$,
Test for overall effect: $Z=2.18(P=0.03)$

Total $(95 \%$ Cl)

$2073169639 \quad 44740 \quad 4348346$

Heterogeneity. Tau $^{2}=0.01 ;$ Chi $^{2}=28.33, d f=16(P=0.03) ; l^{2}=44 \%$

Test for overall effect: $Z=4.32(P<0.0001)$

Test for subgroup differences: $\mathrm{Chi}^{2}=1.35, \mathrm{df}=1(\mathrm{P}=0.25), \mathrm{I}^{2}=25.7 \%$
Mantel-Haenszel, Random, $95 \% \mathrm{Cl}$

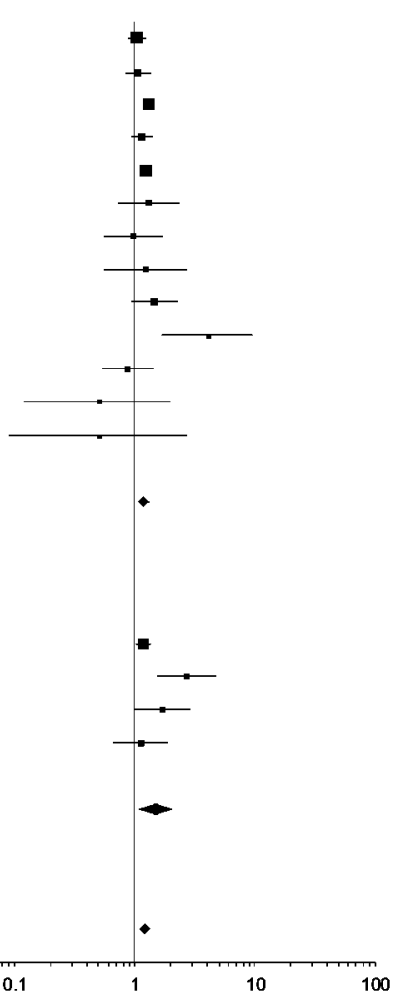




\section{Cohort / Case $\quad$ Comparison}

Study or Subgroup

Events

Events

Total

Odds Ratio

Prospective

Jordan 2016

Berard 2015

Furu 2015

Ban 2014

Louik 2014

Vasilakis-Scaramozza 2013

$\begin{array}{rr}121 & 12962 \\ 89 & 3625 \\ 530 & 34535 \\ 90 & 10401 \\ 447 & 8805 \\ 18 & 3287 \\ 6 & 556 \\ 19 & 2709 \\ 14 & 601\end{array}$

$\begin{array}{rrrr}4503 & 506155 & 11.4 \% & 1.05[0.88,1.26] \\ 344 & 14868 & 8.6 \% & 1.06[0.84,1.35] \\ 26745 & 2266875 & 17.7 \% & 1.31[1.21,1.44] \\ 2556 & 338726 & 9.8 \% & 1.15[0.93,1.42] \\ 370 & 8611 & 13.9 \% & 1.19[1.03,1.37] \\ 28 & 6617 & 2.1 \% & 1.30[0.72,2.35] \\ 541 & 61648 & 1.2 \% & 1.23[0.55,2.77] \\ 512 & 107320 & 201.0 \% & 1.47[0.93,2.33] \\ 8 & 1359 & 1.0 \% & 4.03[1.68,9.65]\end{array}$

$\begin{array}{llll}1334 & 77481 & 35607 & 3312179\end{array}$

Subtotal ( $95 \% \mathrm{Cl})$

Heterogeneity. Tau $^{2}=0.01 ; \mathrm{Chi}^{2}=14.54, \mathrm{df}=8(\mathrm{P}=0.07) ; \mathrm{I}^{2}=45 \%$

Test for overall effect: $Z=3.47(P=0.0005)$

\section{Retrospective}

Huybrechts 2014

Polen 2013

De Jonge 2013

Margulis 2013

Awan 2010

Davis 2007 a

Davis 2007b

Kulin 1998

\section{Subtotal (95\%C)}

$739 \quad 92158$

91331036167

$\begin{array}{rr}580 & 64389 \\ 74 & 15758 \\ 14 & 873 \\ 16 & 3046 \\ 34 & 6853 \\ 17 & 805 \\ 2 & 167 \\ 2 & 267\end{array}$

6403

$14 \quad 8002$

$281 \quad 29223$

$48 \quad 8991$

$26 \quad 5869$

$1171 \quad 49031$

$1186 \quad 49669$

267

Heterogeneity Tau $^{2}=0.05 ; \mathrm{Chi}^{2}=13.73, \mathrm{df}=7(\mathrm{P}=0.06) ; \mathrm{I}^{2}=49 \%$

Test for overall effect: $Z=1.67(P=0.10)$

Total $(95 \%$ Cl)

$2073169639 \quad 44740 \quad 4348346$

Heterogeneity: Tau $^{2}=0.01 ;$ Chi $^{2}=28.33, d f=16(P=0.03) ; l^{2}=44 \%$

Test for overall effect: $Z=4.32(P<0.0001)$

Test for subgroup differences: $\mathrm{Chi}^{2}=0.03, \mathrm{df}=1(\mathrm{P}=0.87), \mathrm{I}^{2}=0 \%$

$(95 \% \mathrm{Cl})$

Odds Ratio
Mantel-Haenszel, Random, $\mathbf{9 5 \%} \mathrm{Cl}$

$68.9 \% \quad 1.21[1.09,1.34]$

$\begin{array}{cc}17.8 \% & 1.25[1.15,1.36] \\ 2.2 \% & 2.69[1.52,4.77] \\ 2.4 \% & 1.68[0.98,2.88] \\ 2.2 \% & 0.98[0.56,1.73] \\ 2.7 \% & 1.12[0.67,1.87] \\ 3.0 \% & 0.88[0.54,1.43] \\ 0.4 \% & 0.50[0.12,2.00] \\ 0.3 \% & 0.50[0.09,2.73]\end{array}$

$31.1 \% \quad 1.24[0.96,1.59]$
$100.0 \% \quad 1.22[1.11,1.33]$

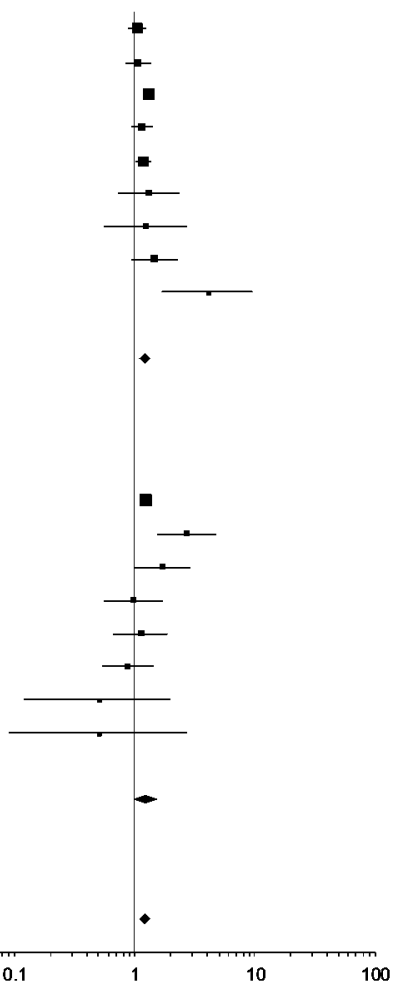

